\title{
Spectroscopy of quadrupole and octupole states in rare-earth nuclei from a Gogny force
}

\author{
K. Nomura, ${ }^{1}$ R. Rodríguez-Guzmán, ${ }^{2}$ and L. M. Robledo ${ }^{3}$ \\ ${ }^{1}$ Grand Accélérateur National d'Ions Lourds, CEA/DSM-CNRS/IN2P3, B.P. 55027, F-14076 Caen Cedex 5, France \\ ${ }^{2}$ Physics Department, Kuwait University, 13060 Kuwait, Kuwait \\ ${ }^{3}$ Departamento de Física Teórica, Universidad Autónoma de Madrid, E-28049 Madrid, Spain \\ (Received 13 April 2015; revised manuscript received 28 May 2015; published 17 July 2015)
}

\begin{abstract}
Collective quadrupole and octupole states are described in a series of Sm and Gd isotopes within the framework of the interacting boson model (IBM), whose Hamiltonian parameters are deduced from mean-field calculations with the Gogny energy density functional. The link between both frameworks is the $\left(\beta_{2} \beta_{3}\right)$ potential energy surface computed within the Hartree-Fock-Bogoliubov framework in the case of the Gogny force. The diagonalization of the IBM Hamiltonian provides excitation energies and transition strengths of an assorted set of states including both positive- and negative-parity states. The resultant spectroscopic properties are compared with the available experimental data and also with the results of the configuration mixing calculations with the Gogny force within the generator coordinate method (GCM). The structure of excited $0^{+}$states and its connection with double-octupole phonons is also addressed. The model is shown to describe the empirical trend of the low-energy quadrupole and octupole collective structure fairly well and turns out to be consistent with GCM results obtained with the Gogny force.
\end{abstract}

DOI: 10.1103/PhysRevC.92.014312

PACS number(s): 21.10.Re, 21.60.Ev, 21.60.Fw, 21.60.Jz

\section{INTRODUCTION}

The study of the equilibrium shapes and the corresponding excitation spectra of atomic nuclei is one of the recurrent themes in nuclear structure physics. Most of the deformed medium-heavy and heavy nuclei exhibit reflection-symmetric ground states. However, in some regions of the nuclear chart, there is an onset of reflection-asymmetric shapes driven by specific shell effects. In quadrupole deformed nuclei, a characteristic feature of octupole deformation is the alternating-parity rotational band formed by the even-spin positive parity states and alternating odd-spin negative-parity states, connected with each other by enhanced electric dipole transitions [1].

In the framework of the spherical shell model, octupolarity arises as a result of the coupling between the $(l, j)$ orbitals in a major shell and the unique-parity $(l+3, j+3)$ intruders from the next major shell. Within this context, illustrative examples are the rare-earth nuclei with the proton number $Z \approx 56$ and the neutron number $N \approx 88$, as well as the light actinides with $Z \approx 88$ and $N \approx 134$. In the light actinides case, the coupling of both neutron (i.e., $1 g_{9 / 2}$ and $0 j_{15 / 2}$ ) and proton (i.e., $1 f_{7 / 2}$ and $\left.0 i_{13 / 2}\right)$ single-particle states leads to octupole deformed ground states [1,2]. A recent Coulomb excitation study has revealed, for the first time, unambiguous evidences of static octupole deformation in ${ }^{224} \mathrm{Ra}$ [3].

In this work, we study the impact of octupole correlations on the ground state and the associated low-lying collective spectra of the nuclei ${ }^{146-156} \mathrm{Sm}$ and ${ }^{148-158} \mathrm{Gd}$. We consider both quadrupole and octupole degrees of freedom. The selected nuclei belong to a region of the nuclear chart where octupole correlations are expected to play an important role and, therefore, represent a valuable testing ground for the considered theoretical approximations. Indeed, the experimental observation of octupole correlations at medium spin, as well as the crossing of the octupole and the ground-state bands, point to the coexistence of reflection symmetric and asymmetric structures in both ${ }^{150} \mathrm{Sm}$ [4] and ${ }^{148} \mathrm{Sm}$ [5]. From the experimental point of view, four low-lying negative-parity bands have already been identified in ${ }^{152} \mathrm{Sm}$ [6]. The emerging pattern of excitations suggests a complex shape coexistence in this nucleus. Moreover, the nucleus ${ }^{152} \mathrm{Sm}$ has been identified [7] as an example of the $\mathrm{X}(5)$ critical point symmetry [8]. The nature of many low-lying excited $0^{+}$states in rare-earth nuclei has also attracted much attention. For example, 13 excited $0^{+}$states have already been identified for ${ }^{158} \mathrm{Gd}$ [9]. Within the $s p d f$-IBM framework (where IBM stands for interacting boson model), many of the observed $0^{+}$states have been attributed to the coupling of two octupole phonons [10].

Keeping in mind the experimental findings mentioned above, it is interesting and timely to consider a systematic analysis of the quadrupole-octupole collectivity in rare-earth nuclei. The breaking of reflection symmetry and the associated low-lying negative-parity states have been addressed using various theoretical frameworks: self-consistent mean-field [11-20], algebraic [21-25], collective phenomenological [26-33], and cluster [34-36] models. A large number of calculations for nuclei with static and/or dynamical octupole deformations have already been reported [12,14-20,37-39]. In particular, the nuclear energy density functional (EDF) framework, both at the mean-field level and beyond, provides a reasonably accurate description of the properties of the negative- and positive-parity states all over the nuclear chart [40]. Both nonrelativistic [41-43] and relativistic [44,45] EDFs have already been applied in both mean-field and beyond-mean-field studies of medium-heavy- and heavy-mass nuclei. The description of the excitation spectra and transition rates requires the inclusion of dynamical (i.e., beyond meanfield) correlations associated with the restoration of the broken symmetries and/or fluctuations in the collective parameters (i.e., generating coordinates) $[19,20,40,46]$. Within this context, the projection of the intrinsic (i.e., symmetry-broken) states onto good-parity ones as well as the corresponding 
configuration mixing, in the spirit of the two-dimensional generator coordinate method (GCM) [47], have been considered recently for nuclei in the rare-earth region using the quadrupole $Q_{20}$ and octupole $Q_{30}$ moments as generating coordinates [19]. For recent GCM study, based on $Q_{30}$-constrained mean-field states, the reader is also referred to Ref. [48].

In this work we first carry out $\left(Q_{20}, Q_{30}\right)$-constrained Hartree-Fock-Bogoliubov (HFB) calculations based on the Gogny-EDF [43]. Such calculations provide us with the corresponding (axially symmetric) mean-field potential energy surfaces (PESs). Subsequently, to obtain the spectrum and wave functions of the excited states, we employ the interacting boson model (IBM) [49]. The essence of our method is to determine the parameters of an appropriate IBM Hamiltonian by calculating the associated bosonic PES so that it matches the Gogny-HFB PES. The IBM Hamiltonian resulting from our fermion-to-boson mapping procedure is then used in spectroscopic calculations. A similar mapping has been used in previous studies of low-lying quadrupole states [50-52] and shape coexistence [53]. Recently, the method [49] has been extended to describe quadrupole-octupole correlations and shape transitions in the light actinide and rare-earth regions [54,55] based on the relativistic DD-PC1 EDF.

The same Gogny-EDF can be used along with beyondmean-field techniques to restore the broken reflection symmetry and compute the properties of the lowest-lying negative-parity state. The excitation energy and transition strengths, when compared with the IBM numbers, can be used as a benchmark to test the consistency of the mapping procedure. Therefore, one of the goals of this study is to assess the fermion-to-boson mapping methodology in the description of spectroscopic properties in rare-earth nuclei. We compare the IBM spectra and transition rates with previous Gogny-GCM calculations for the same Sm and Gd nuclei [19], as well as with available experimental data. Here we also refer the reader to the previous IBM study based on the relativistic mean-field (RMF) approximation [55]. We have used the D1M [56] parametrization of the Gogny-EDF, which was originally designed to better describe nuclear masses. It has been shown [19,57-60] that the D1M parameter set essentially retains the same predictive power as the standard and thoroughly tested Gogny-D1S [61] one. We have also performed a selected set of calculations based on the D1S parametrization to examine the robustness of our predictions with respect to the particular version of the Gogny-EDF employed. However, as the corresponding HFB [19] and IBM results are quite similar, in the present paper we only focus on calculations based on the D1M parameter set.

The paper is organized as follows. In Sec. II, we briefly outline the HFB-to-IBM mapping procedure. Next, in Sec. III, we discuss the systematics of the $\left(\beta_{20}, \beta_{30}\right)$ [62] PESs obtained for the considered nuclei, as well as the parameters of the IBM Hamiltonian. The results of the spectroscopic calculations are discussed in Sec. IV. First, in Sec. IV A, we present the systematics of the low-energy spectra and the reduced transition probabilities in ${ }^{146-156} \mathrm{Sm}$ and ${ }^{148-158} \mathrm{Gd}$. We compare with available experimental data as well as with results obtained within the Gogny-GCM approximation [19]. Next, in Sec. IV B we further illustrate the predictive power of the mapped IBM model with a detailed discussion of the spectroscopic properties for ${ }^{150} \mathrm{Sm}$ (a soft nucleus along the quadrupole and octupole directions) and ${ }^{158} \mathrm{Gd}$ (a strongly quadrupole deformed nucleus). To obtain some insight into the nature of the excited $0^{+}$states in the studied nuclei, their systematics is discussed in Sec. IV C. In Sec. IV D, we discuss the IBM correlation energies and compare them with Gogny-GCM results. Finally, Sec. V is devoted to some concluding remarks and work perspectives.

\section{FRAMEWORK}

In this section we briefly outline the HFB-to-IBM mapping scheme [55]. Our starting point is a set of axially symmetric $\left(Q_{20}, Q_{30}\right)$-constrained Gogny-HFB calculations [19]. They provide us with the corresponding mean-field potential energy surfaces (MFPESs) and the HFB states $\left|\Phi\left(Q_{20}, Q_{30}\right)\right\rangle$ for the nuclei ${ }^{146-156} \mathrm{Sm}$ and ${ }^{148-158} \mathrm{Gd}$. For simplicity, both the quadrupole $Q_{20}$ and the octupole $Q_{30}$ moments are then translated into the standard $\beta_{2}$ and $\beta_{3}$ mean-field deformation parameters.

Subsequently, the MFPESs obtained are mapped into their bosonic counterparts, i.e., the IBM potential energy surfaces (IBMPESs). This procedure allows us to determine the parameters of the IBM Hamiltonian used in the spectroscopic calculations. The IBM Hamiltonian is converted into a PES by means of a set of coherent bosonic states and this IBMPES is what is used to match the Gogny-HFB PES [55]. Note that the MFPESs correspond to the total HFB energies; i.e., neither mass parameters nor zero-point (rotational and/or vibrational) quantum corrections are included.

The description of the quadrupole and octupole deformations as well as the positive- and negative-parity states within the IBM framework requires both positive- and negative-parity bosons. Here one assumes that the low-lying positive-parity states are reasonably well described by the pairs of valence nucleons associated with the $s$ and $d$ bosons, respectively. However, negative-parity states are assumed to be described by the coupling to octupole $f$ bosons [63]. Therefore, our entire IBM model space comprises the $s, d$, and $f$ bosons. For simplicity, we do not distinguish between proton and neutron bosons. A more complete description of the low-energy collective states would require the inclusion of the dipole $p$ boson that could be associated with the spurious center-ofmass motion [22] or to the giant dipole resonance [64]. This, however, lies out of the scope of the present paper and is left for future work.

The $s d f$ Hamiltonian used is given by

$$
\hat{H}=\epsilon_{d} \hat{n}_{d}+\epsilon_{f} \hat{n}_{f}+\kappa_{2} \hat{Q}_{2} \cdot \hat{Q}_{2}+\kappa_{2}^{\prime} \hat{L}_{d} \cdot \hat{L}_{d}+\kappa_{3} \hat{Q}_{3} \cdot \hat{Q}_{3},
$$

where the first (second) term stands for the number operator for the $d(f)$ bosons with $\epsilon_{d}\left(\epsilon_{f}\right)$ being the single $d(f)$ boson energy relative to the $s$ boson one. The third term represents the quadrupole-quadrupole interaction with strength $\kappa_{2}$. The quadrupole operator is given as

$$
\hat{Q}_{2}=s^{\dagger} \tilde{d}+d^{\dagger} \tilde{s}+\chi_{d d}\left[d^{\dagger} \times \tilde{d}\right]^{(2)}+\chi_{f f}\left[f^{\dagger} \times \tilde{f}\right]^{(2)},
$$

where $\chi_{d d}$ and $\chi_{f f}$ are parameters. The fourth term in Eq. (1) is the rotational one relevant for the $s d$ space. In this case, the 
angular momentum operator $\hat{L}_{d}$ reads

$$
\hat{L}_{d}=\sqrt{10}\left[d^{\dagger} \times \tilde{d}\right]^{(1)} .
$$

The last term in Eq. (1) is the octupole-octupole interaction with the strength parameter $\kappa_{3}$. The octupole operator takes the form

$$
\hat{Q}_{3}=s^{\dagger} \tilde{f}+f^{\dagger} \tilde{s}+\chi_{d f}\left[d^{\dagger} \times \tilde{f}+f^{\dagger} \times \tilde{d}\right]^{(2)},
$$

with $\chi_{d f}$ being a parameter.

Note that Eq. (1) does not represent the most general form for the $s d f$ Hamiltonian. The present form has already been used in previous phenomenological IBM studies which have confirmed its suitability to describe the available experimental data. The Hamiltonian $\hat{H}^{\mathrm{IBM}}$ of Eq. (1) can be derived from a microscopic octupole-octupole interaction between proton and neutron bosons by mapping the totally symmetric state in the IBM-2 space onto the equivalent one in the IBM-1 space [65]. We neglect the dipole-dipole interaction term $\hat{L}_{d} \cdot \hat{L}_{f}$ (with $\left.\hat{L}_{f}=\sqrt{28}\left[d^{\dagger} \times \tilde{f}\right]^{(1)}\right)$, because it has been shown [25] to be of little relevance for low-energy states.

The IBMPES is calculated as the expectation value of the Hamiltonian Eq. (1) in the boson condensate state $|\phi\rangle$ [66]

$$
|\phi\rangle=\frac{1}{\sqrt{N_{B}}}\left(\lambda^{\dagger}\right)^{N_{B}}|-\rangle \quad \text { with } \quad \lambda^{\dagger}=s^{\dagger}+\bar{\beta}_{2} d_{0}^{\dagger}+\bar{\beta}_{3} f_{0}^{\dagger},
$$

where $N_{B}\left(=n_{s}+n_{d}+n_{f}\right)$ and $|-\rangle$ denote the total number of bosons (i.e., half the number of valence nucleons [63]) and the inert core, respectively. In the present study, the doubly magic nucleus ${ }^{132} \mathrm{Sn}$ is assumed to be the inert core. Therefore, $N_{B}$ runs from 6 to 12 (7 to 13) in ${ }^{146-156} \mathrm{Sm}\left({ }^{148-158} \mathrm{Gd}\right)$. For the quadrupole case $(\lambda=2)$ the bosonic $\bar{\beta}_{2}$ and fermionic $\beta_{2}$ deformations can be related as $\tilde{\beta}_{2}=C_{2} \beta_{2}$ [66], with $C_{2}$ being a coefficient. Here, as in previous works [54,55], we assume that $\tilde{\beta}_{3}=C_{3} \beta_{3}$, with $C_{3}$ being an additional coefficient.

To reduce the computational effort, it has been customary in many of the previous phenomenological IBM calculations to restrict the maximum number of $f$ bosons to $n_{f}^{\max }=1$ in the diagonalization of the IBM Hamiltonian. However, as shown in the next section, the microscopic PESs may exhibit a sizable ground-state octupole deformation, which requires a larger number of $f$ bosons in our IBM calculations. Therefore, both positive- and negative-parity bosons are treated on an equal footing. As a consequence, a truncation on $n_{f}^{\max }$ is not used and the number of $f$ bosons can run from 0 to $N_{B}$. This also holds true for the $s$ and $d$ bosons. Let us also mention that previous phenomenological studies (e.g., [67,68]) have also suggested the need for more negative-parity bosons for a better description of the experimental data.

The analytic IBMPES reads

$$
\begin{aligned}
E\left(\bar{\beta}_{2}, \bar{\beta}_{3}\right)= & \frac{N_{B}}{1+\bar{\beta}_{2}^{2}+\bar{\beta}_{3}^{2}}\left(\epsilon_{s}^{\prime}+\epsilon_{d}^{\prime} \bar{\beta}_{2}^{2}+\epsilon_{f}^{\prime} \bar{\beta}_{3}^{2}\right)+\frac{N_{B}\left(N_{B}-1\right)}{\left(1+\bar{\beta}_{2}^{2}+\bar{\beta}_{3}^{2}\right)^{2}} \\
& \times\left[\kappa_{2}\left(2 \bar{\beta}_{2}-\sqrt{\frac{2}{7}} \chi_{d d} \bar{\beta}_{2}^{2}-\frac{2}{\sqrt{21}} \chi_{f f} \bar{\beta}_{3}^{2}\right)^{2}\right. \\
& \left.-4 \kappa_{3}\left(\bar{\beta}_{3}-\frac{2}{\sqrt{15}} \chi_{d f} \bar{\beta}_{2} \bar{\beta}_{3}\right)^{2}\right]
\end{aligned}
$$

with

$$
\begin{aligned}
\epsilon_{s}^{\prime}=5 \kappa_{2}-7 \kappa_{3}, \quad \epsilon_{d}^{\prime} & =\epsilon_{d}+6 \kappa_{2}^{\prime}+\left(1+\chi_{d d}^{2}\right) \kappa_{2}-\frac{7}{5} \chi_{d f}^{2} \kappa_{3}, \\
\text { and } \quad \epsilon_{f}^{\prime} & =\epsilon_{f}-\frac{5}{7} \chi_{f f}^{2} \kappa_{2}+\left(1+\chi_{d f}^{2}\right) \kappa_{3} .
\end{aligned}
$$

The IBMPES $E\left(\bar{\beta}_{2}, \bar{\beta}_{3}\right)$ is specified by the parameters of the Hamiltonian in Eq. (1) plus the coefficients $C_{2}$ and $C_{3}$. We have determined those parameters by fitting the IBMPESs to the Gogny-D1M MFPESs using the same procedure as in Ref. [50]. Let us remark that, even though a simplified Hamiltonian Eq. (1) is considered, there is still a larger number of parameters to be determined, as compared to the $s d$-IBM system. Therefore, rather than trying to fit all the parameters at once, we first determine the ones relevant for the $s d$ space $\left(\epsilon_{d}, \kappa_{2}, \chi_{d d}, C_{2}\right.$, and $\left.\kappa_{2}^{\prime}\right)$ and then those associated with the $f$ space as well as the ones associated with the coupling between the two spaces $\left(\epsilon_{f}, \kappa_{3}, \chi_{f f}, \chi_{d f}\right.$, and $\left.C_{3}\right)$. The $\hat{L}_{d} \cdot \hat{L}_{d}$ term in Eq. (1) does not contribute to the PESs, and therefore its strength $\kappa_{2}^{\prime}$ is determined independently by comparing the fermionic and bosonic cranking moment of inertia (see Ref. [51] for details). The (fermionic) Thouless-Valatin [69] moment of inertia for the $2_{1}^{+}$state reads

$$
\mathcal{I}_{\mathrm{TV}}=3 / E_{\gamma} .
$$

where $E_{\gamma}$ stands for the $2_{1}^{+}$excitation energy obtained from the self-consistent cranking calculation with the constraint $\left\langle\hat{J}_{x}\right\rangle=\sqrt{J(J+1)}$, where $\hat{J}_{x}$ represents the $x$ component of the angular momentum operator. However, the IBM moment of inertia is computed using the coherent state $|\phi(\beta, \gamma)\rangle$ and the Schaaser-Brink [70] expression

$$
\mathcal{I}_{\mathrm{IBM}}=\lim _{\omega \rightarrow \infty} \frac{1}{\omega} \frac{\left\langle\phi(\beta, \gamma)\left|\hat{L}_{x}\right| \phi(\beta, \gamma)\right\rangle}{\langle\phi(\beta, \gamma) \mid \phi(\beta, \gamma)\rangle}
$$

with $\omega$ being the cranking frequency.

Having the parameters $\epsilon_{d}^{\prime}\left(=\epsilon_{d}-6 \kappa_{2}^{\prime}\right), \kappa_{2}, \chi_{d d}$, and $C_{2}$ already determined from the fit of the IBMPES to the MFPES in the $s d$ space, the IBM moment of inertia in Eq. (9) depends only in the parameter $\kappa_{2}^{\prime}$, whose value is determined so that $\mathcal{I}_{\mathrm{IBM}}$ is equal to the $\mathcal{I}_{\mathrm{TV}}$ value at the energy minimum.

From the diagonalization of the $s d f$-IBM Hamiltonian, we have obtained both the energies and the wave functions of the spectrum, which are labeled by total spin and parity quantum numbers. We have used the computer program OCTUPOLE [71]. The reduced electromagnetic transition probabilities $B\left(E \lambda ; J \rightarrow J^{\prime}\right)=\left|\left\langle J^{\prime}|| \hat{T}^{(E \lambda)}|| J\right\rangle\right|^{2} /(2 J+1) \quad(\lambda=$ $1,2,3)$ are then computed using the resulting IBM wave functions. Here $J\left(J^{\prime}\right)$ denotes the spin for the initial (final) state. Of particular interest for the present study are the dipole $E$ 1, quadrupole $E 2$, and octupole $E 3$ transition probabilities defined in terms of the operators

$$
\begin{aligned}
& T^{(E 1)}=e_{1}\left[d^{\dagger} \times \tilde{f}+d^{\dagger} \times \tilde{f}\right]^{(1)}, \\
& T^{(E 2)}=e_{2} \hat{Q}_{2}, \\
& T^{(E 3)}=e_{3} \hat{Q}_{3},
\end{aligned}
$$

where $\hat{Q}_{2}$ and $\hat{Q}_{3}$ are the quadrupole and octupole operators, respectively, appearing in the IBM Hamiltonian and $e_{\lambda}$ 's are boson effective charges which are kept constant for all 
the considered nuclei. Their values are taken from previous phenomenological IBM studies $\left(e_{1}=0.01 e \mathrm{~b}^{1 / 2}[68], e_{2}=\right.$ $0.13 e \mathrm{~b}$ [68], and $e_{3}=0.099 \mathrm{eb}^{3 / 2}$ [23]). It has been shown that they provide a reasonable overall description of the experimental data. However, they are not the ones derived microscopically. Therefore, in the following discussions, one should always keep in mind that there is some extra freedom in the overall scale of the calculated IBM transitions.

\section{MEAN-FIELD POTENTIAL ENERGY SURFACES AND THE PARAMETERS OF THE IBM HAMILTONIAN}

In this section, we discuss the systematics of the MFPESs and IBMPESs as well as the parameters of the IBM Hamiltonian obtained along the lines described in Sec. II.

The axially symmetric Gogny-D1M MFPESs are shown in Fig. 1 for ${ }^{146-156} \mathrm{Sm}$ and ${ }^{148-158} \mathrm{Gd}$. The MFPESs of some of the $\mathrm{Sm}$ isotopes have already been presented in Ref. [19] as illustrative examples. However, for the sake of completeness, in the figure we have included all the MFPESs both for Sm and $\mathrm{Gd}$ nuclei. For the sake of presentation, the plots in the figure correspond to $-0.3 \leqslant \beta_{2} \leqslant 0.5$ and $0.0 \leqslant \beta_{3} \leqslant 0.2$, as well as to an energy range of $5 \mathrm{MeV}$ from the absolute minimum. We have tested that the previous ranges are enough to describe the considered low-energy collective states and used them to build our IBM Hamiltonian.

A spherical reflection-symmetric ground state is predicted for the nuclei ${ }^{146} \mathrm{Sm}$ [panel (a)] and ${ }^{148} \mathrm{Gd}$ [panel (g)], respectively. However, the MFPESs become soft for isotopes with neutron numbers $N=86$ and 88 , indicating that the
Gogny-HFB approximation can only be considered as a valuable starting point in such nuclei but beyond-mean-field correlations should be taken into account [19]. Moreover, the $N=88$ isotopes exhibit the softest MFPESs with a shallow minimum at a nonzero $\beta_{3}$ value. One also sees that the MFPESs become steeper along the $\beta_{3}$ direction for isotopes with $N \geqslant 90$. Similar trends have been found up to $N=88$ in previous RMF calculations [37,55], based on the EDFs PK1 [72] and DD-PC1 [73], respectively. However, in those calculations, the octupole minima are more pronounced than ours. In fact, the previous study with the relativistic functional DD-PC1 [55] suggested that the PES is much softer along the $\beta_{3}$ direction. The same trend was found for isotopes with $N \geqslant 90$.

As discussed in Ref. [19], there is no essential difference between the overall topology of the MFPESs obtained with the Gogny-D1M and Gogny-D1S EDFs. However, at a quantitative level, the latter provides MFPESs with slightly deeper absolute minima than the former. Nevertheless, such a difference turns out to be too small to significantly affect either the IBM parameters or the energies and wave functions of the excited states. With this in mind, in what follows only results based on the Gogny-D1M EDF are discussed.

In Fig. 2 we have depicted the (mapped) IBMPESs. First, we observe that they are much flatter than the HFB MFPESs (see Fig. 1). This is a common feature of the IBM framework already found in previous studies $[49,50]$. The reason is that IBM's model space is rather limited and only comprises pairs of valence nucleons. This leads to flat IBMPESs for larger deformations. However, one should keep in mind that

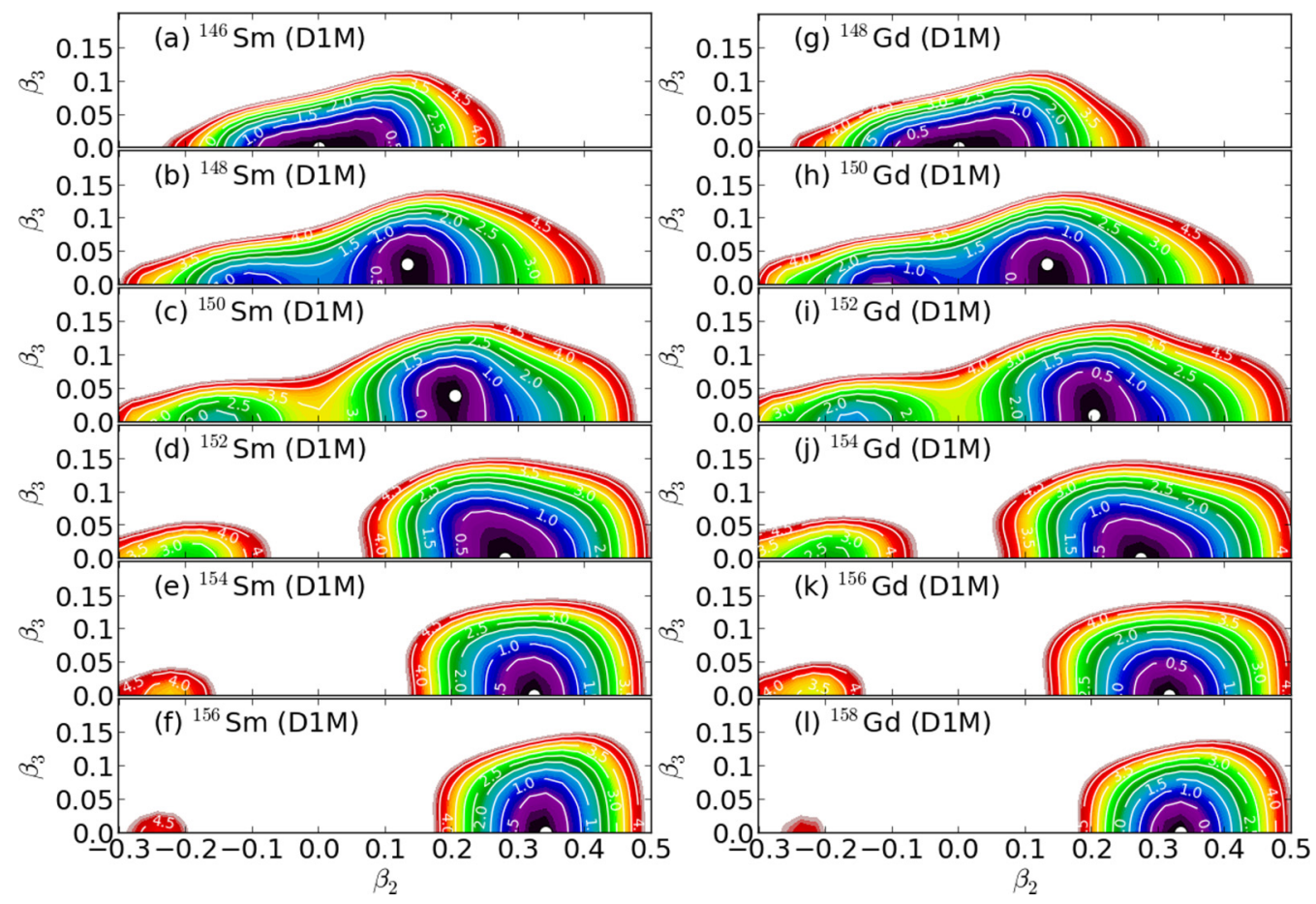

FIG. 1. (Color online) Axially symmetric $\left(\beta_{2}, \beta_{3}\right)$ PESs for the nuclei ${ }^{146-156} \mathrm{Sm}$ and ${ }^{148-158}$ Gd calculated within the constrained Gogny-HFB approach based on the D1M parametrization. The contour lines join points with the same energy (in MeV) and the color scale varies in steps of $100 \mathrm{keV}$. The energy difference between neighboring contours is $0.5 \mathrm{MeV}$. These $\left(\beta_{2}, \beta_{3}\right)$ energy surfaces are symmetric with respect to the $\beta_{3}=0$ axis. Thus, they are only plotted for $\beta_{3} \geqslant 0$. For each nucleus the absolute minimum is identified by an open circle. 

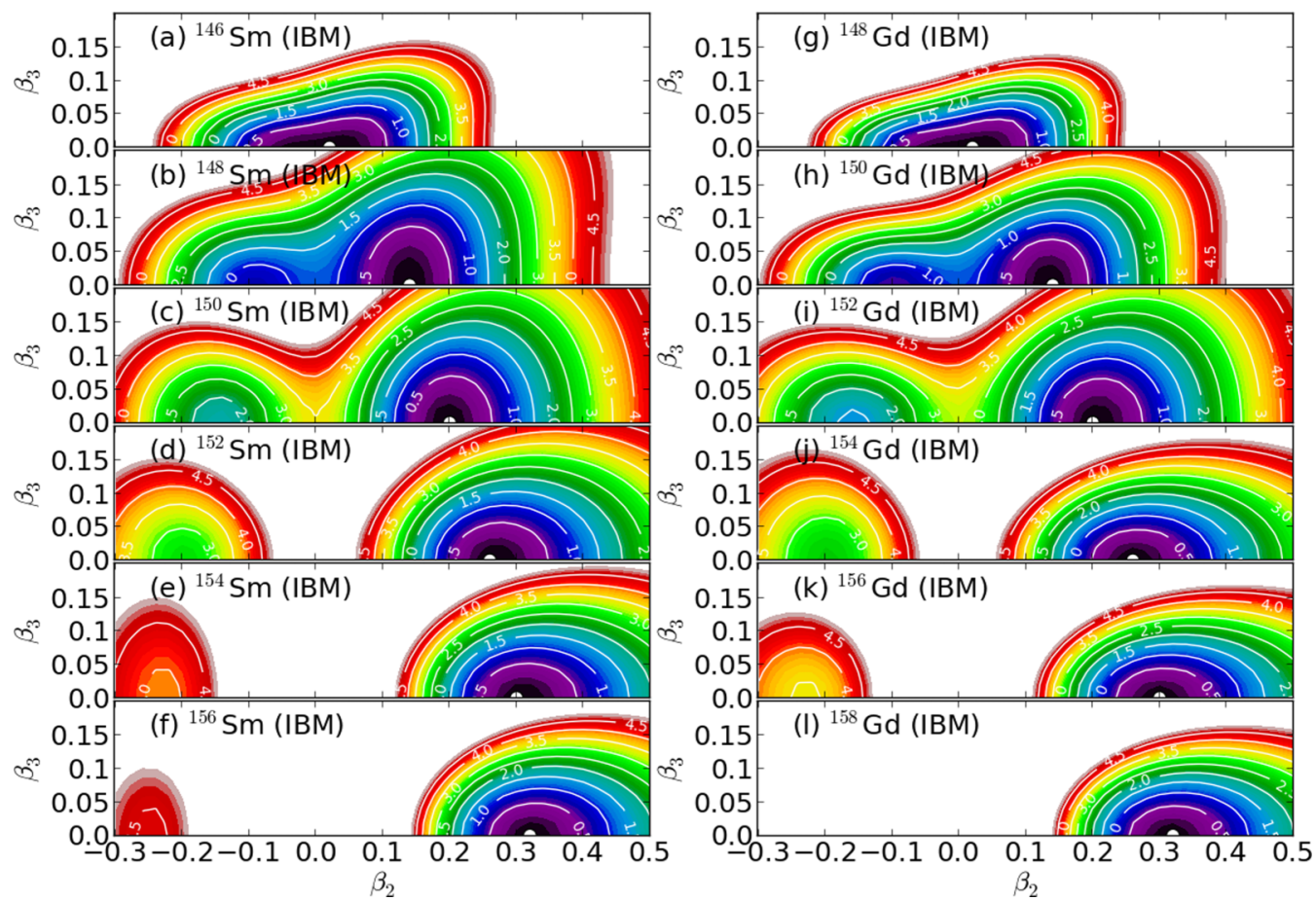

FIG. 2. (Color online) The same as Fig. 1 but for the mapped IBM PESs.

within the considered fermion-to-boson mapping, the topology far away from the absolute minimum is not relevant as long as we restrict our analysis to the low-lying collective states. Hence, we only focus on reproducing the curvatures of the Gogny-D1M MFPESs in the neighborhood (a 5-MeV window) of the absolute minimum, along both the $\beta_{2}$ and $\beta_{3}$ directions.

Second, we note that, for $N=86$ and 88 isotopes, the MFPES predicts a shallow absolute minimum at nonzero $\beta_{3}$ values (Fig. 1), while in the corresponding IBMPES the absolute minimum is found at $\beta_{3}=0$ (Fig. 2). However, as the depth of this absolute minimum in the MFPESs differs by at most tens of keVs from the saddle point on the $\beta_{3}=0$ axis, we assume that the discrepancy of the absolute minimum point, which is not deep enough in energy, between the MFPES and the IBMPES is not of crucial importance for the final result.

Bearing those in mind, the IBMPESs in Fig. 2 closely follow, for each of the considered nuclei, the basic topology as well as the overall systematic trend of the Gogny-HFB ones shown in Fig. 1.

In Fig. 3, the IBM parameters for the considered Sm and Gd nuclei are plotted as functions of neutron number. As can be observed in panels (a) and (b), the single $d\left(\epsilon_{d}\right)$ and $f$ $\left(\epsilon_{f}\right)$ boson energies decrease as functions of neutron number. From a microscopic point of view, as already discussed in the context of the $s d$-IBM-2 [63,74,75] model, the decrease of $\epsilon_{d}$ could be related to the coupling of the unperturbed $d$ boson with other types of bosons not yet explicitly included in the model space. Alternatively, when one derives the form of the IBM Hamiltonian in Eq. (1) from a general $s d f$-IBM Hamiltonian, several two-body terms of the general IBM Hamiltonian, which are reduced to the kinetic energies of $d$ and $f$ bosons multiplied with the boson-number-dependent factors, are absorbed in $\epsilon_{d}$ and $\epsilon_{f}$, thereby making the parameters vary significantly with boson number [76].

The coupling strength of the quadrupole-quadrupole interaction $\kappa_{2}$, shown in panel (c), is almost constant. A similar trend has been found in the IBM study based on the RMF approximation [55]. A sudden change is observed in the parameter $\chi_{f f}$, plotted in panel (d), around $N=88$ and is correlated with the significant change observed in the MFPESs (see Fig. 1). However, at variance with our previous $s d$-IBM study in the same mass region [50], the parameter $\chi_{d d}$ [panel (e)] is rather constant. Compared to the quadrupole-quadrupole coupling $\kappa_{2}$ [panel (c)], the strength of the octupole-octupole interaction $\kappa_{3}$ [panel (f)] exhibits a gradual decrease with increasing neutron number.

In panel $(\mathrm{g})$ of the same figure, we have plotted the strength $\kappa_{2}^{\prime}$ of the $\hat{L}_{d} \cdot \hat{L}_{d}$ term Eq. (1). Its negative value, for all the studied nuclei, leads to the lowering of the positive-parity yrast states [51]. Note that $\kappa_{2}^{\prime}$ is not considered for the spherical nuclei ${ }^{146} \mathrm{Sm}$ and ${ }^{148} \mathrm{Gd}$. As shown below, the experimental spectra for these nuclei do not exhibit a rotational-like structure and, therefore, there is no obvious reason for introducing the $\hat{L}_{d} \cdot \hat{L}_{d}$ term in the corresponding calculations. The parameters $\chi_{d f}$ [panel (h)] exhibit a pronounced isotopic dependence with a maximum around $N=88-90$ which correlates well with the octupole softness of the MFPESs around the same neutron numbers. Both the $C_{2}$ [panel (i)] and the $C_{3}$ [panel (j)] coefficients change smoothly with neutron number [55].

\section{SPECTROSCOPIC CALCULATIONS}

In this section, we discuss the results of the calculations with the IBM Hamiltonian for ${ }^{146-156} \mathrm{Sm}$ and ${ }^{148-158} \mathrm{Gd}$. First, in Sec. IV A, the systematics of the low-energy spectra and the 

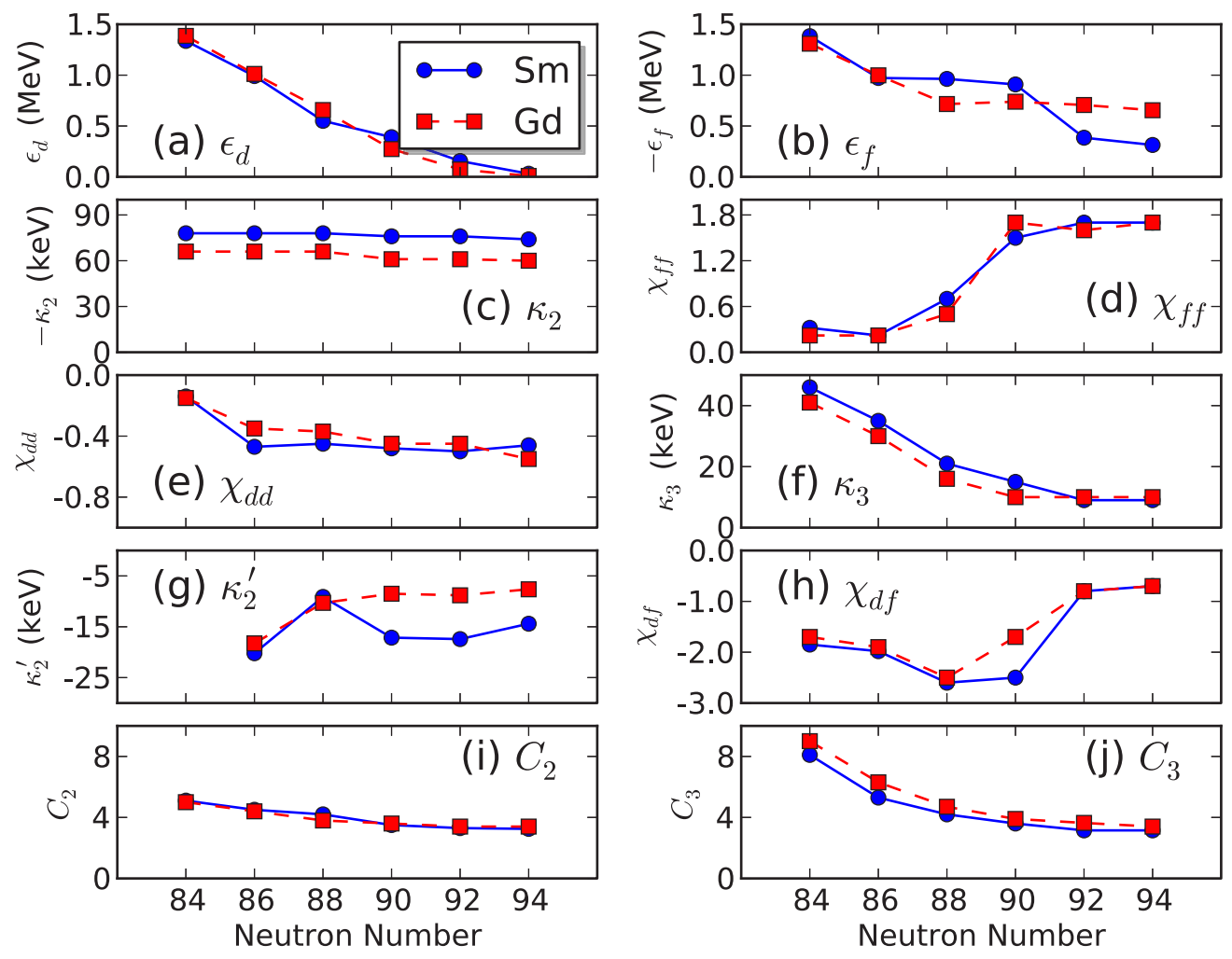

FIG. 3. (Color online) The parameters of the $s d f$-IBM Hamiltonian $\hat{H}$ in Eq. (1), as well as the proportionality coefficients $C_{2}$ and $C_{3}$, are plotted as functions of the neutron number for the considered nuclei. The parameters $\chi_{d d}, \chi_{f f}, \chi_{d f}, C_{2}$, and $C_{3}$ are are dimensionless.

reduced transition probabilities in ${ }^{146-156} \mathrm{Sm}$ and ${ }^{148-158} \mathrm{Gd}$ is addressed. Next, in Sec. IV B, the spectroscopic properties predicted for the nuclei ${ }^{150} \mathrm{Sm}$ and ${ }^{158} \mathrm{Gd}$ are discussed in detail. The systematics of the excited $0^{+}$states is presented in Sec. IV C. Finally, in Sec. IV D, ground-state correlation energies are discussed.

\section{A. Systematics of the low-energy spectra and the reduced transition probabilities in ${ }^{146-156} \mathrm{Sm}$ and ${ }^{148-158} \mathrm{Gd}$}

In Figs. 4 and 5 the low-energy positive- and negative-parity yrast states, as calculated with the mapped $s d f$-IBM Hamiltonian are plotted for the nuclei ${ }^{146-156} \mathrm{Sm}$ and ${ }^{148-158} \mathrm{Gd}$. The theoretical results are compared with the available experimental data taken from the NNDC compilation [77]. Because our predictions for Sm [panels (a) and (b)] and Gd [panels (c) and (d)] isotopes are rather similar, we mainly discuss the former.

The lowering of the energies with increasing neutron number $N$ is consistent with a shape transition (see Fig. 1) to a strongly quadrupole deformed configurations. Indeed, the ratios $R_{4 / 2} \equiv E\left(4_{1}^{+}\right) / E\left(2_{1}^{+}\right)=2.33$ and 2.38 obtained for ${ }^{146,148} \mathrm{Sm}$ are both close to the vibrational limit, while the theoretical (experimental) $R_{4 / 2}$ values for the transitional nuclei ${ }^{150,152} \mathrm{Sm}$ are $2.82(2.31)$ and 2.91 (3.01), respectively. Our calculations predict a more pronounced rotational character for ${ }^{150} \mathrm{Sm}$ than expected from the experiment. However, it is remarkable that the $R_{4 / 2}$ value for the ${ }^{152} \mathrm{Sm}$ is exactly the same as the $\mathrm{X}(5)$ one [8]. For the heavier isotopes, our IBM calculations predict well-developed rotational bands. For example, in

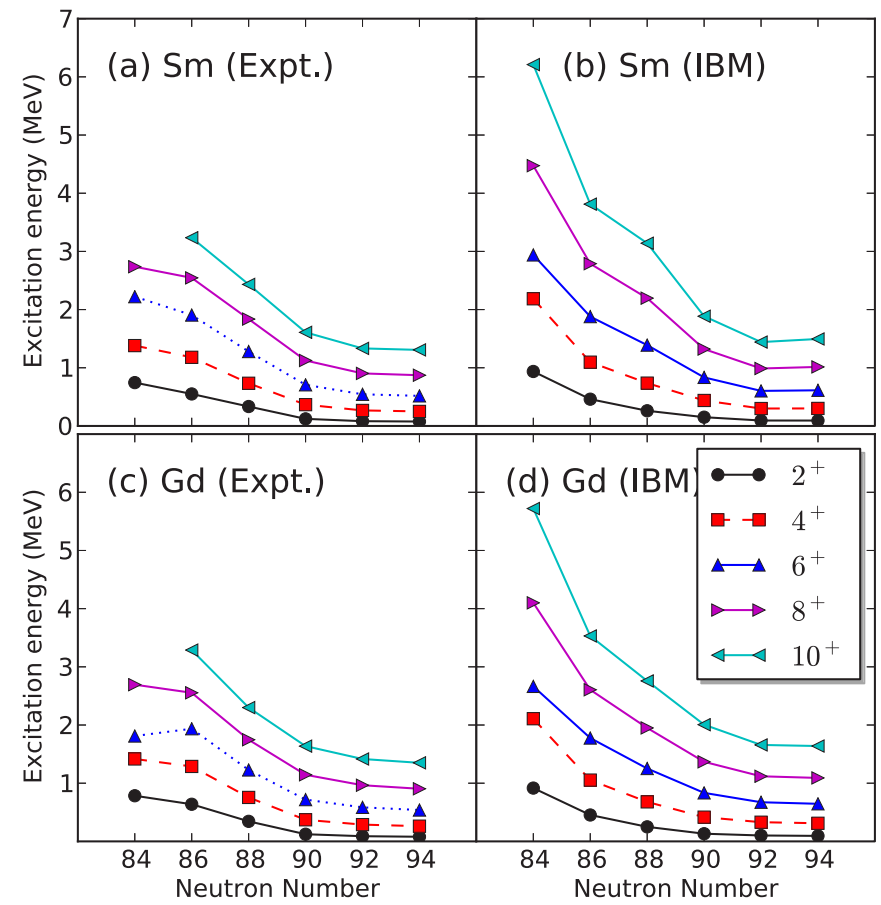

FIG. 4. (Color online) The energy spectra of the lowest-lying even-spin positive-parity states up to $J^{\pi}=10^{+}$for the considered $\mathrm{Sm}$ and $\mathrm{Gd}$ isotopes. All the experimental data are taken from the NNDC compilation [77]. 


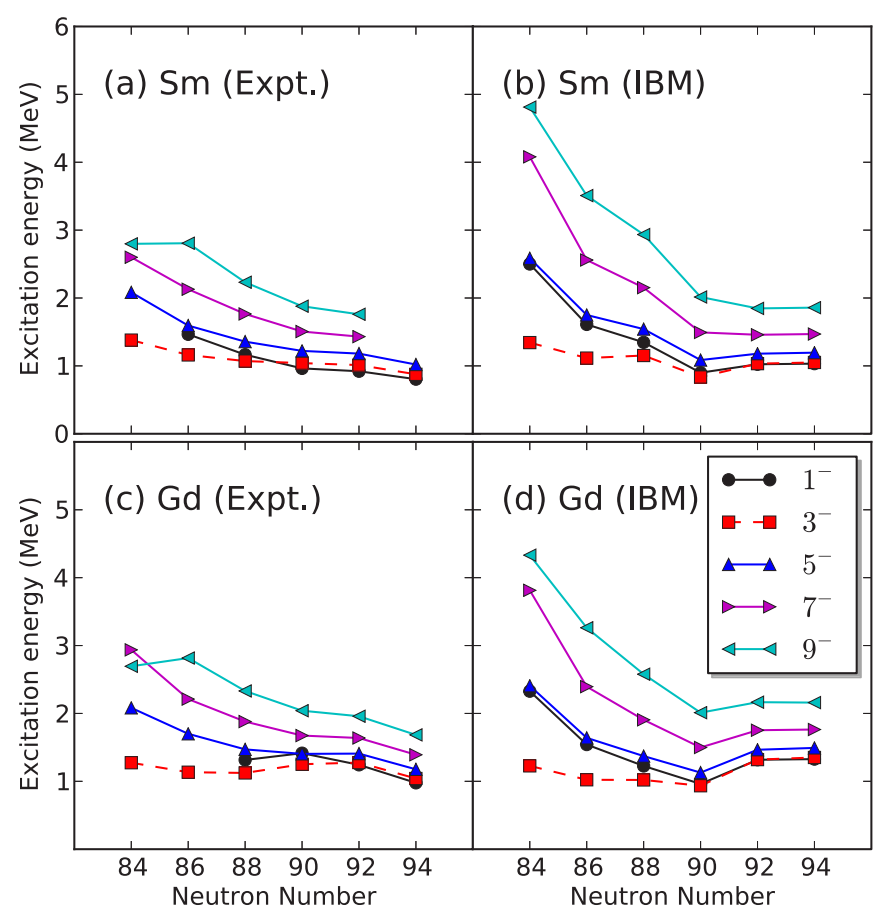

FIG. 5. (Color online) The same as in Fig. 4, but for the lowestlying odd-spin negative-parity states up to $J^{\pi}=9^{-}$.

the case of ${ }^{154,156} \mathrm{Sm}$, we have obtained the ratios $R_{4 / 2}=3.21$ and 3.25, respectively. The theoretical results agree reasonably well with the experimental ones except for the lightest isotopes where the energies of the higher spin states are overestimated. The reason for the overestimation could be the too-restricted model space and/or Hamiltonian of the IBM that is not rich enough to reproduce the peculiar topology of the Gogny-EDF MFPES for the lightest isotopes. We recall that the $\hat{L}_{d} \cdot \hat{L}_{d}$ term is not included in ${ }^{146} \mathrm{Sm}$ and ${ }^{148} \mathrm{Gd}$ because it is of little importance for these spherical nuclei [51]. One could introduce this term phenomenologically to fix the overestimation, which is, however, out of scope of the present work.

The $J^{\pi}=1^{-}, \ldots, 9^{-}$states, plotted in Fig. 5, display features characteristic of the octupole collectivity. With an exception made for the $3^{-}$states, their excitation energies decrease sharply for $84 \leqslant N \leqslant 90$. At variance with the experimental data, the theoretical excitation energies increase for $N>90$, which correlates well with the diminishing of the octupole minimum depth observed in the MFPESs (see Fig. 1). In both isotopic chains, the $3_{1}^{-}$state is lower in energy than the $1_{1}^{-}$one. We have also found a near degeneracy for the $1_{1}^{-}$and the $5_{1}^{-}$states for $N \leqslant 88-90$. This octupole vibrational feature becomes more apparent for the lighter isotopes.

In Fig. 6, we have compared the excitation energies of the lowest $1_{1}^{-}$states with the ones obtained in the framework of a two-dimensional GCM calculations [19] also with the GognyD1M EDF. The predicted IBM and GCM values are quite similar for $84 \leqslant N \leqslant 88$. In the case of the $\mathrm{Sm}$ isotopes both the GCM and IBM excitation energies increase with increasing neutron number though the former exhibit a more pronounced change than the latter. Similar results are obtained for $\mathrm{Gd}$ isotopes, with an exception made for the fact that the smallest

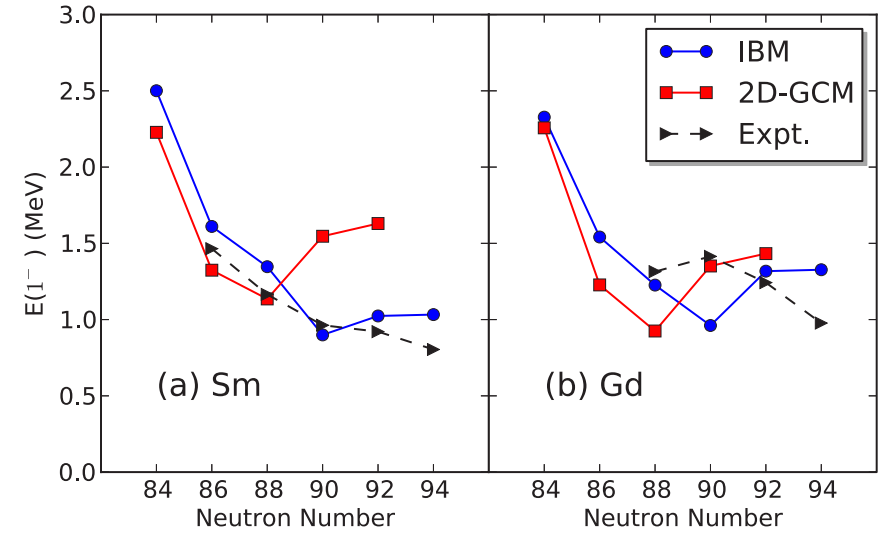

FIG. 6. (Color online) The excitation energies of the $1_{1}^{-}$states predicted with the mapped IBM Hamiltonian are compared with the ones obtained in the framework of two-dimensional GCM calculations [19] for Sm (a) and Gd (b) nuclei. In both methods, the Gogny-D1M parametrization has been used. The experimental energy levels are also included in the figure.

$1^{-}$excitation energy is found at $N=88(N=90)$ in the GCM (IBM) calculations.

We have studied the quantity

$$
S(J)=\frac{[E(J+1)-E(J)]-[E(J)-E(J-1)]}{E\left(2_{1}^{+}\right)},
$$

which is sensitive to the splitting between the positive- and negative-parity members of a rotational band. In Eq. (13), $E(J)$ stands for the excitation energy of the $J=0^{+}, 1^{-}, 2^{+}, 3^{-}, \ldots$ state. Note that, for an ideal alternating-parity band, we would obtain an equal energy splitting between the positive- and negative-parity states differing by $\Delta J=1$. This, in turn, would lead to $S(J) \approx 0$. However, a nonzero $S(J)$ value indicates a deviation from a pure alternating-parity band.

In Fig. 7 we have plotted $S(J)$, as a function of the spin $J$, for ${ }^{150,152,156} \mathrm{Sm}$ which are taken as representative examples. The experimental data for ${ }^{150} \mathrm{Sm}$ [panel (a)] oscillate with $J$

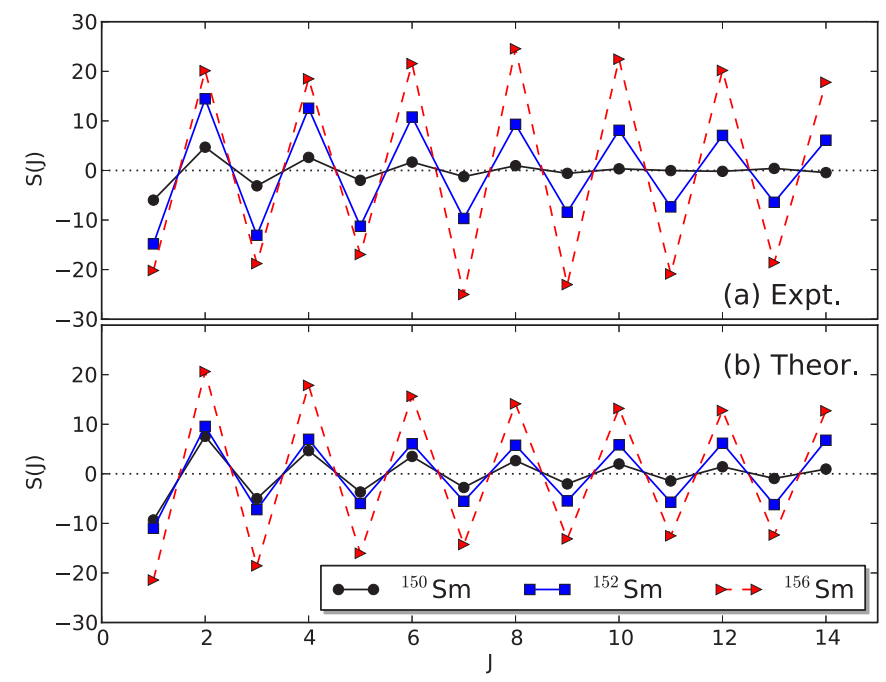

FIG. 7. (Color online) Signature splitting $S(J)$ of ${ }^{150,152,156} \mathrm{Sm}$ nuclei as a function of spin $J$. For more details, see the main text. 


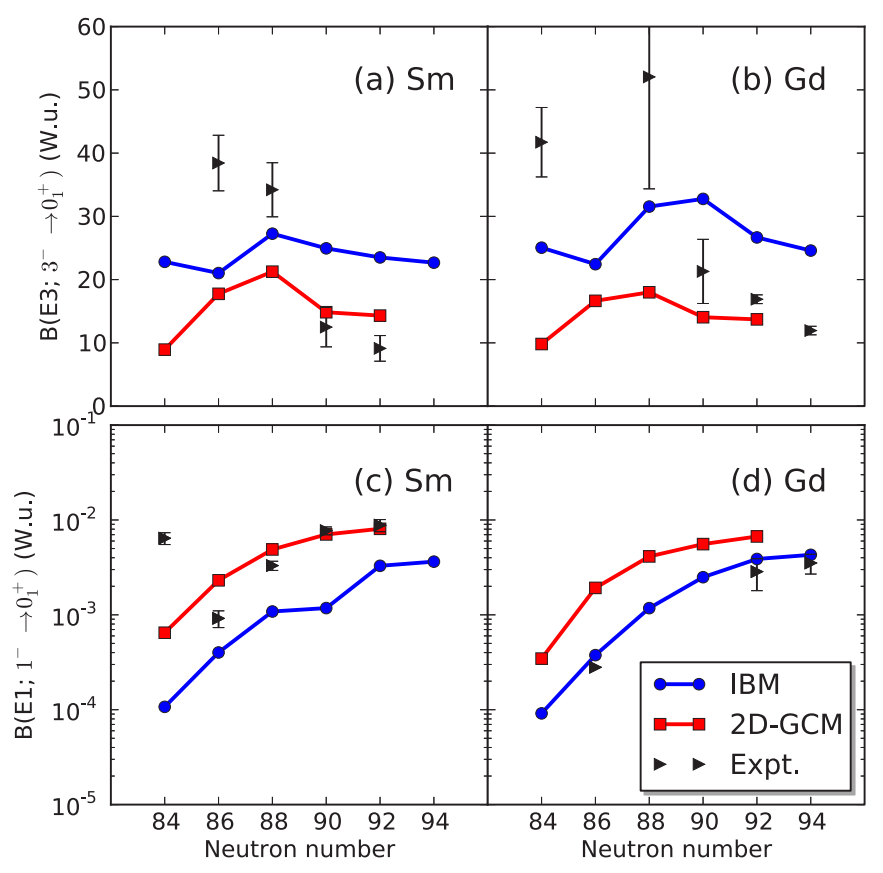

FIG. 8. (Color online) Theoretical and experimental transition probabilities $B\left(E 3 ; 3_{1}^{-} \rightarrow 0_{1}^{+}\right)$and $B\left(E 1 ; 1_{1}^{-} \rightarrow 0_{1}^{+}\right)$for ${ }^{146-156} \mathrm{Sm}$ and ${ }^{148-158} \mathrm{Gd}$. The experimental data are taken from Refs. [77-80].

but become zero around $J \approx 8^{+}$. Though larger deviations are observed in our calculations [panel (b)], their global trend resembles the experimental one. Both theoretically and experimentally, the deviation from $S(J)=0$ in ${ }^{156} \mathrm{Sm}$ is more pronounced than for ${ }^{150,152} \mathrm{Sm}$. This suggests a deviation from the ideal alternating-parity band behavior and also correlates well with the behavior of the Gogny-D1M MFPESs (see Fig. 1).
The reduced transition probabilities $B\left(E 3 ; 3_{1}^{-} \rightarrow 0_{1}^{+}\right)$and $B\left(E 1 ; 1_{1}^{-} \rightarrow 0_{1}^{+}\right)$are compared in Fig. 8 with the experimental data [77-80]. For both isotopic chains, the predicted E3 transition rates [panels (a) and (b)] exhibit a weak dependence on the neutron number with a maximum at $N=88-90$. The down-sloping tendency in the theoretical (IBM) E3 values observed in the heavier isotopes is consistent with the experiment, though a smoother change with neutron number is found for $\mathrm{Sm}$ isotopes. However, the $E 1$ transition rates [panels (c) and (d)] increase with increasing neutron number which agrees quite well with the experiment, with an exception made for ${ }^{146} \mathrm{Sm}$. The overall trend also agrees well with the one found in previous IBM [55] and GCM [19] calculations. Note that the discrepancy of the IBM rates with the experimental ones are partly a consequence of the particular choice of the IBM effective charges. No effective charges are needed within the GCM framework [19] as all the nucleons are considered in the wave functions.

\section{B. Spectroscopy of the nuclei ${ }^{150} \mathrm{Sm}$ and ${ }^{158} \mathrm{Gd}$}

The low-lying spectrum of ${ }^{150} \mathrm{Sm}$ is compared in Fig. 9 with the available experimental excitation energies [77]. The band assignment has been made according to the dominant $E 2$ transition sequence. The IBM energies are generally more stretched than the experimental ones. Approximate alternating parity bands can be seen with the level ordering $7^{-}, 8^{+}, 9^{-}$, $10^{+}, \ldots$, etc.

A noticeable deviation with respect to the experimental data is obtained for the $\beta$-vibrational bandhead. In fact, the experimental excitation energy of this $0_{2}^{+}$state is as small as the one for the $4_{1}^{+}$state. However, in the calculations it is almost twice higher, suggesting a too-limited IBM model space. On the other hand, for the quasi- $\gamma$ band, with the $K^{\pi}=2^{+}$built on the $2_{2}^{+}$state, our calculations predict the staggering $\left(3_{\gamma}^{+}, 4_{\gamma}^{+}\right)$,

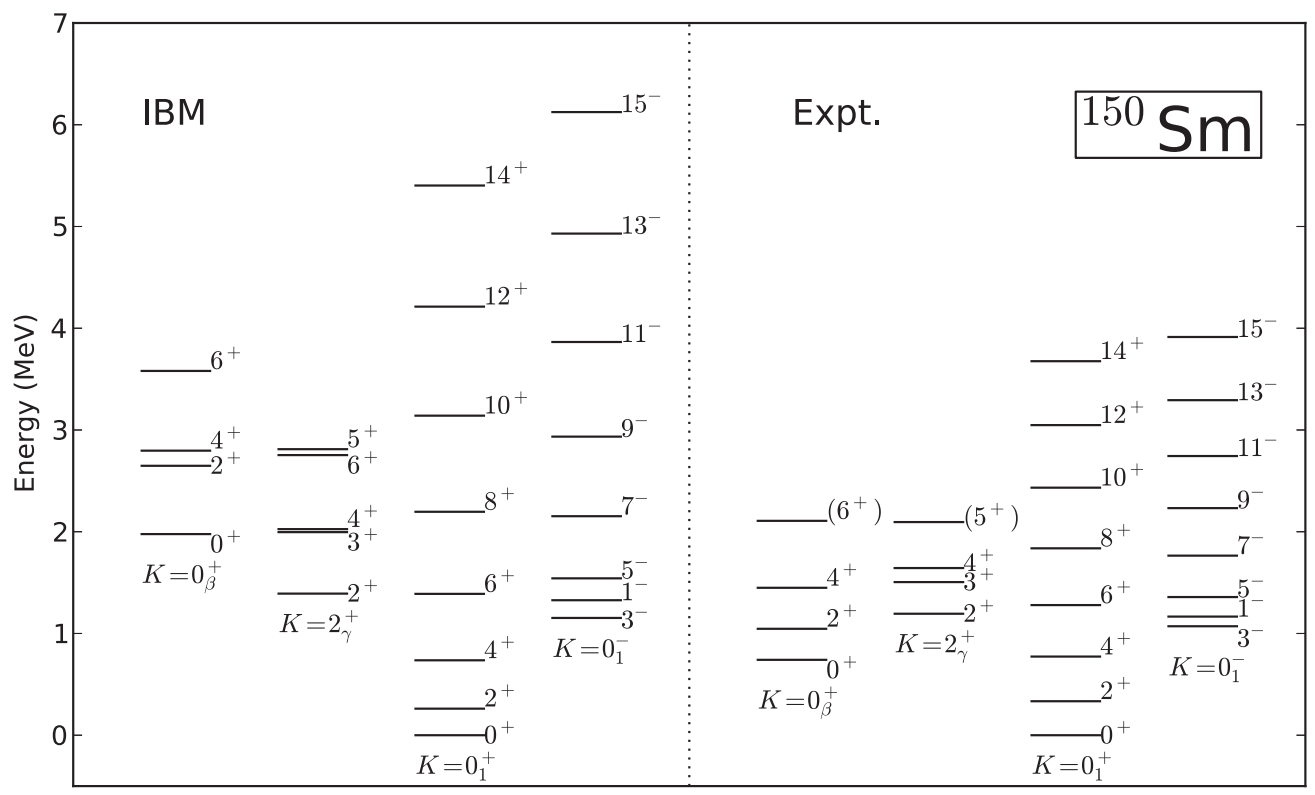

FIG. 9. Comparison of the low-energy spectrum predicted within the IBM framework for the nucleus ${ }^{150}$ Sm with the available experimental excitation energies [77]. 
TABLE I. Theoretical and experimental [77] $B(\mathrm{E} 2)$ transitions for ${ }^{150} \mathrm{Sm}$ (in Weisskopf units). For details, see the main text.

\begin{tabular}{|c|c|c|c|}
\hline$J_{i}^{\pi}$ & $J_{f}^{\pi}$ & $B(E 2)_{\text {theor. }}$ & $B(E 2)_{\text {expt. }}$ \\
\hline $2_{1}^{+}$ & $0_{1}^{+}$ & 79 & $57.1(13)$ \\
\hline $4_{1}^{+}$ & $2_{1}^{+}$ & 112 & $110(17)$ \\
\hline $6_{1}^{+}$ & $4_{1}^{+}$ & 120 & $1.5 \times 10^{+2}(5)$ \\
\hline $8_{1}^{+}$ & $6_{1}^{+}$ & 117 & $1.7 \times 10^{+2}(9)$ \\
\hline $0_{\beta}^{+}$ & $2_{1}^{+}$ & 10 & $53(5)$ \\
\hline \multirow[t]{4}{*}{$2_{\beta}^{+}$} & $0_{1}^{+}$ & 1.78 & $0.81_{-21}^{+26}$ \\
\hline & $0_{\beta}^{+}$ & 24 & $1.1 \times 10_{-3}^{2+4}$ \\
\hline & $2_{1}^{+}$ & 0.038 & - \\
\hline & $4_{1}^{+}$ & 3.92 & - \\
\hline \multirow[t]{5}{*}{$2_{\gamma}^{+}$} & $0_{1}^{+}$ & 1.18 & $2.1(15)$ \\
\hline & $0_{\beta}^{+}$ & 10.2 & $9.1(24)$ \\
\hline & $2_{1}^{+}$ & 21 & - \\
\hline & $2_{\beta}^{+}$ & 4.09 & - \\
\hline & $4_{1}^{+}$ & 0.064 & $7(3)$ \\
\hline $3_{1}^{+}$ & $2_{\beta}^{+}$ & 11.1 & - \\
\hline \multirow[t]{4}{*}{$4_{\beta}^{+}$} & $2_{1}^{+}$ & 0.86 & - \\
\hline & $2_{\beta}^{+}$ & 11.2 & $1.9 \times 10^{+2}(9)$ \\
\hline & $2_{\gamma}^{+}$ & 0.039 & $42(20)$ \\
\hline & $3_{1}^{+}$ & 0.34 & - \\
\hline \multirow[t]{3}{*}{$4_{\gamma}^{+}$} & $2_{1}^{+}$ & 0.14 & $1.4(7)$ \\
\hline & $2_{\beta}^{+}$ & 2.1 & $4.1(21)$ \\
\hline & $2_{\gamma}^{+}$ & 42 & - \\
\hline $1_{1}^{-}$ & $3_{K=0^{-}}^{-}$ & 109 & - \\
\hline $5_{K=0^{-}}^{-}$ & $3_{K=0^{-}}^{--0}$ & 70 & - \\
\hline $7_{K=0^{-}}^{--}$ & $5_{K=0^{-}}^{--}$ & 84 & - \\
\hline
\end{tabular}

$\left(5_{\gamma}^{+}, 6_{\gamma}^{+}\right)$, etc. This reflects the lack of triaxiality in the present study. The inclusion of mean-field triaxiality as well as the relevant terms in the mapped IBM Hamiltonian could be useful to better describe the structure of the quasi- $\gamma$ band [52]. Work along these lines is in progress and will be reported elsewhere.

The $E 2$ and $E 1$ transition rates obtained for ${ }^{150} \mathrm{Sm}$ are compared with the experimental ones [77] in Tables I and II, respectively. Most of the predicted $E 2$ values agree reasonably well with those from the experiment. Note that our calculations account for the $K=0^{-}$band, built on the $3_{1}^{-}$state, with strong $E 2$ transitions. Nevertheless, large discrepancies are also found for some interband transitions. For example, the $0_{\beta}^{+} \rightarrow 2_{1}^{+}$strength is considerably underestimated. Stronger interband $E 2$ transitions suggest a significant mixing between different intrinsic configurations. Indeed, a recent experiment has suggested a complex shape coexistence in ${ }^{152} \mathrm{Sm}$ [6]. Within this context, an IBM model space larger than the one considered in the present study may be required. A configuration mixing associated with intruder states [53] could also be introduced to better describe a transitional nucleus like ${ }^{150} \mathrm{Sm}$. Another alternative could be the inclusion of triaxiality to better constrain the form of the IBM Hamiltonian. Furthermore, the value $B\left(E 2 ; 4_{\beta}^{+} \rightarrow 2_{\gamma}^{+}\right)=0.039$ W.u. is too small as compared with the experimental one [42(20) W.u]. A possible reason may be that the $0_{\beta}^{+}$states as well as the ones built on it might not be well described by the present calculations.
TABLE II. Same as in Table I, but for the $E 1$ transitions (in $10^{-3}$ W.u.).

\begin{tabular}{lccc}
\hline \hline$J_{i}^{\pi}$ & $J_{f}^{\pi}$ & $B(E 1)_{\text {theor. }}$ & $B(E 1)_{\text {expt }}$ \\
\hline $1_{K=0^{-}}^{-}$ & $0_{1}^{+}$ & 1.1 & $1.4_{-5}^{+7}$ \\
$3_{K=0^{-}}^{-}$ & $2_{1}^{+}$ & 0.13 & $2.9_{-10}^{+14}$ \\
$4_{\beta}^{+}$ & $2_{1}^{+}$ & 2.5 & $5_{-3}^{+4}$ \\
& $4_{1}^{+}$ & $1.8 \times 10^{-4}$ & $5_{-3}^{+4}$ \\
$4_{\gamma}^{+}$ & $3_{K=0^{-}}^{-4}$ & 2.6 & - \\
& $5_{K=0^{-}}^{-}$ & 0.24 & - \\
$5_{K=0^{-}}^{-}$ & $3_{K=0^{-}}^{-}$ & 0.087 & $0.27(13)$ \\
$6_{1}^{+}$ & $5_{K=0^{-}}^{-}$ & 0.54 & $0.9(5)$ \\
$7_{K=0^{-}}^{-}$ & $4_{1}^{+}$ & 4.2 & - \\
$8_{1}^{+}$ & $5_{K=0^{-}}^{-}$ & 0.027 & - \\
$9_{K=0^{-}}^{-}$ & $6_{1}^{+}$ & 5.8 & - \\
$10_{1}^{+}$ & $7_{K=0^{-}}^{-}$ & 0.15 & - \\
$11_{K=0^{-}}^{-}$ & $8_{1}^{+}$ & 7.3 & - \\
\hline \hline
\end{tabular}

The calculated $B(E 1)$ values in Table II reveal rather strong transitions (starting around the $J>5^{-}$) from the states of odd- $J$ negative-parity $K=0_{1}^{-}$to those of the even- $(J-1)$ positive-parity ground-state bands. This fact, as well as the increasing $B\left(E 1 ; J_{K=0^{-}}^{-} \rightarrow(J-1)_{K=0_{1}^{+}}^{+}\right)$value, as a function of $J$, signals the existence of an alternating parity band in ${ }^{150} \mathrm{Sm}$. Nevertheless, we do not consider the $B(E 1)$ value obtained in the present calculation to be conclusive, mainly because of the lack of the $p$-boson effect in our framework. Indeed, as already pointed out in previous phenomenological [67] and microscopic [81] studies on octupole-deformed nuclei, the description of these $E 1$ transitions in the IBM framework could be improved by explicitly including the $p$ boson in the model space or by extending the form of the $E 1$ operator so as to absorb the $p$-boson effect in the $s d f$ space.

The low-lying spectrum of ${ }^{158} \mathrm{Gd}$, shown in Fig. 10, exhibits an overall agreement with the available experimental data for the lowest-lying positive- and negative-parity bands. The $1_{1}^{-}$state of the lowest negative-parity band is assigned as the bandhead of the $K^{\pi}=0_{1}^{-}$and the $K^{\pi}=1_{1}^{-}$bands in the present calculation and in the NNDC compilation [77], respectively.

In our calculations, the two lowest-lying positive-parity, $K^{\pi}=0_{1}^{+}$and $2_{\gamma}^{+}$, bands are composed of states with $n_{f} \approx$ 0.02 and $0.06 \leqslant n_{f} \leqslant 0.09$, respectively, suggesting that they are almost pure positive-parity bands. However, the states in the band built on the $0_{2}^{+}\left(0_{\beta}^{+}\right)$state are of two- $f$ boson (equivalently double-octupole phonon) nature with $\left\langle\hat{n}_{f}\right\rangle \approx 2$. The side-band energies, especially for those states in the positive-parity $\beta$-vibrational and quasi- $\gamma$ bands, are overestimated considerably, for similar reasons as in the ${ }^{150} \mathrm{Sm}$ case.

In Tables III and IV, we have compared some relevant $E 2$ and $E 1$ transition rates with the experimental ones [77]. Many of the calculated $E 2$ transition rates agree well with the data. Again, a noticeable deviation is observed for the $4_{\beta}^{+} \rightarrow$ $2_{\gamma}^{+}$transitions, probably for the same reason as in the ${ }^{150} \mathrm{Sm}$ 


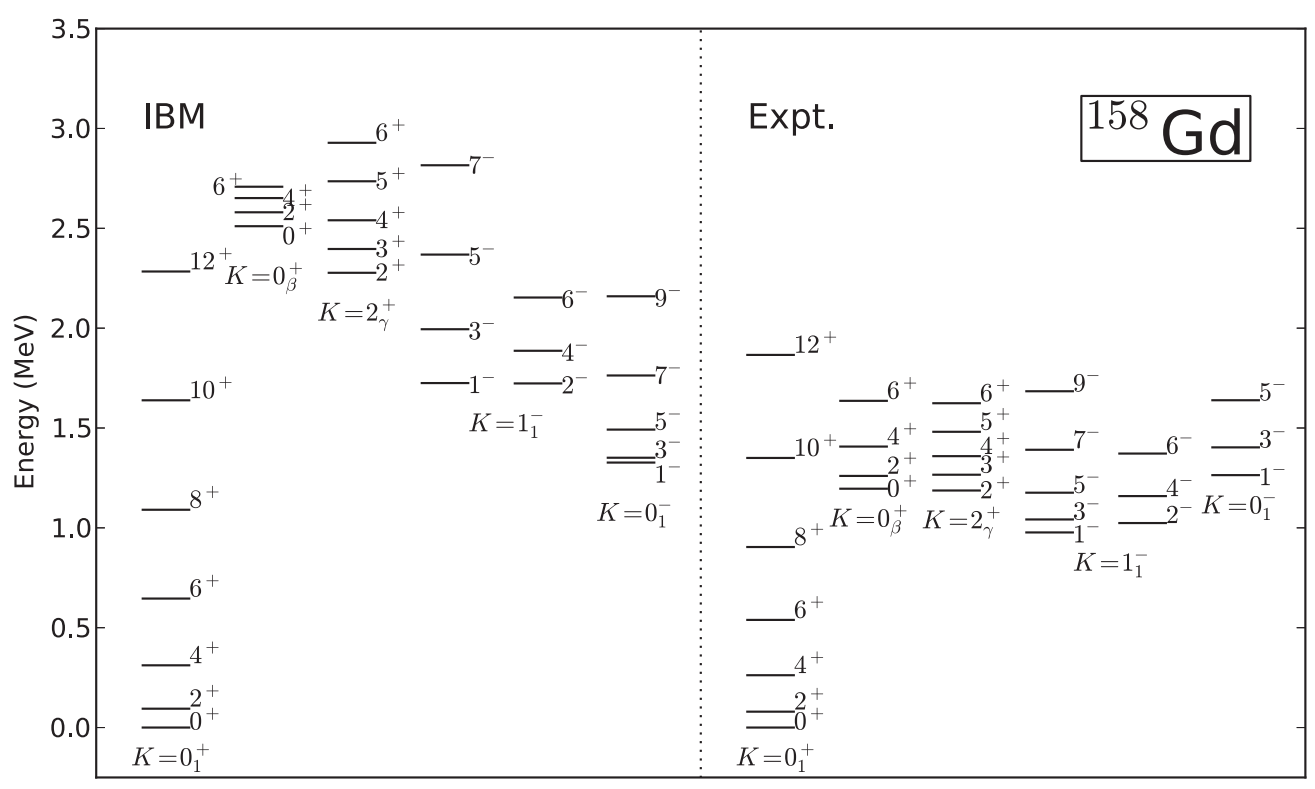

FIG. 10. Same as in Fig. 9 but for the nucleus ${ }^{158} \mathrm{Gd}$.

case. We note that the lifetime of the experimental $3_{K=1^{-}}^{-}$state adapted in Ref. [77] has nearly $25 \%$ uncertainty and that, for that reason, the error bars for the reduced $E 2$ transitions $4_{K=1^{-}}^{-} \rightarrow 3_{K=1^{-}}^{-}$and $5_{K=1^{-}}^{-} \rightarrow 3_{K=1^{-}}^{-}$shown in Ref. [77], as well as in Table III, could be corrected. From Table IV one concludes that our model gives a reasonable description of the $E 1$ transitions associated with states in the $K^{\pi}=0_{1}^{-}$band whose energies are described rather nicely as well (see Fig. 10). However, our model does not account for the $E 1$ transitions associated with the $K^{\pi}=1_{1}^{-}$band.

\section{Excited 0 ${ }^{+}$states}

Experimentally, many excited $0^{+}$states have been identified in the low-energy excitation spectrum of ${ }^{158} \mathrm{Gd}$. The

TABLE III. Same as in Table I but for the nucleus ${ }^{158} \mathrm{Gd}$.

\begin{tabular}{lccc}
\hline \hline$J_{i}^{\pi}$ & $J_{f}^{\pi}$ & $B(E 2)_{\text {theor. }}$ & $B(E 2)_{\text {expt. }}$ \\
\hline $2_{1}^{+}$ & $0_{1}^{+}$ & 170 & $198(6)$ \\
$4_{1}^{+}$ & $2_{1}^{+}$ & 241 & $289(5)$ \\
$6_{1}^{+}$ & $4_{1}^{+}$ & 260 & - \\
$8_{1}^{+}$ & $6_{1}^{+}$ & 264 & $3.3 \times 10^{+2}(3)$ \\
$2_{\gamma}^{+}$ & $0_{1}^{+}$ & 3.9 & $3.4(3)$ \\
& $2_{1}^{+}$ & 7.7 & $6.0(7)$ \\
& $4_{1}^{+}$ & 0.59 & $0.27(4)$ \\
$2_{\beta}^{+}$ & $0_{1}^{+}$ & 0.14 & $0.31(4)$ \\
& $2_{1}^{+}$ & 0.088 & $1.39(15)$ \\
$4_{\beta}^{+}$ & $2_{\gamma}^{+}$ & 0.88 & 12.8 \\
& $2_{\beta}^{+}$ & 93 & - \\
$3_{K=1^{-}}^{-}$ & $1_{K=1^{-}}^{-}$ & 105 & $>1.6 \times 10^{+3}$ \\
$3_{K=0^{-}}^{-}$ & $1_{K=0^{-}}^{-}$ & 146 & $781(14)$ \\
$4_{K=1^{-}}^{-}$ & $3_{K=1^{-}}^{-}$ & 41 & $369(6)$ \\
$5_{K=1^{-}}^{-}$ & $3_{K=1^{-}}^{-}$ & 189 & - \\
$5_{K=0^{-}}^{-}$ & $3_{K=0^{-}}^{-}$ & 159 & $2.09 \times 10^{+3}(3)$ \\
$4_{K=1^{-}}^{-}$ & $2_{K=1^{-}}^{-}$ & 142 & \\
\hline \hline
\end{tabular}

previous phenomenological calculation within the $s p d f$-IBM model [10] showed that such a large number of excited $0^{+}$ states at relatively low energy can be described if the octupole degrees of freedom are taken into account, and many of the $0^{+}$states have been attributed to the coupling of two-octupole phonons. Meanwhile, the emergence of a large number of low-energy excited $0^{+}$states can be a good signature of a quantum phase transition [82].

To address the nature of the $0^{+}$states resulting from the mapped $s d f$-IBM Hamiltonian, we show in Fig. 11 the energy distribution (or level scheme) of the lowest $150^{+}$states and the corresponding average values of the $f$-boson number operator $\left\langle\hat{n}_{f}\right\rangle$ for the ${ }^{146-156} \mathrm{Sm}$ [from panel (a) to panel (f)] and ${ }^{148-158} \mathrm{Gd}$ [from panel (g) to panel (1)] nuclei. In the ${ }^{146} \mathrm{Sm}$ [panel (a)] and ${ }^{148} \mathrm{Gd}$ [panel (g)] cases those states with an energy higher than $8 \mathrm{MeV}$ are not shown. In all the nuclei, the $\mathrm{O}^{+}$ground state is predominantly composed of positive-parity ( $s$ and $d$ ) bosons as $\left\langle\hat{n}_{f}\right\rangle<0.5$. In both isotopic chains, for many of the nuclei with $N \geqslant 90,\left\langle\hat{n}_{f}\right\rangle \approx 2$ for the $0_{2}^{+}$ state, suggesting its double-octupole phonon nature. Moreover,

TABLE IV. Same as in Table I but for the $E 1$ transitions (in units of $10^{-3}$ W.u.) in ${ }^{158} \mathrm{Gd}$.

\begin{tabular}{llcc}
\hline \hline$J_{i}^{\pi}$ & $J_{f}^{\pi}$ & $B(E 1)_{\text {theor. }}$ & $B(E 1)_{\text {expt. }}$ \\
\hline $1_{K=1^{-}}^{-}$ & $0_{1}^{+}$ & 1.5 & $0.098443(4)$ \\
$1_{K=0^{-}}^{-}$ & $2_{1}^{+}$ & 2.8 & $0.096515(6)$ \\
$3_{K=1^{-}}^{-}$ & $0_{1}^{+}$ & 4.3 & $3.5(12)$ \\
& $2_{1}^{+}$ & 2.3 & $6.4(21)$ \\
$3_{K=0^{-}}^{-}$ & $2_{1}^{+}$ & 0.35 & $0.33(10)$ \\
$2_{K=1^{-}}^{-}$ & $4_{1}^{+}$ & 3.4 & $0.29(8)$ \\
$4_{K=1^{-}}^{-}$ & $2_{1}^{+}$ & 6.8 & $>1.1$ \\
\hline \hline
\end{tabular}



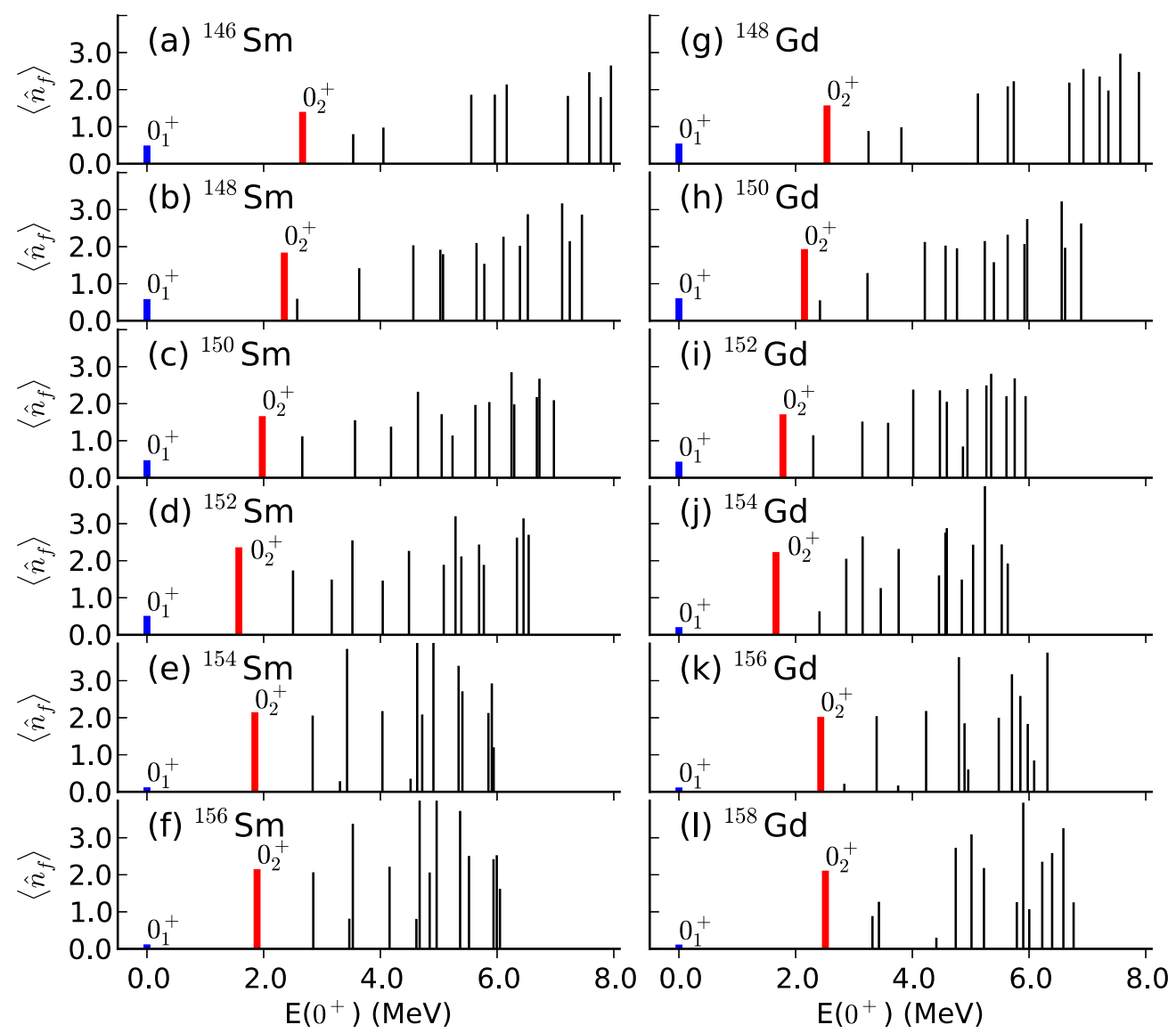

FIG. 11. (Color online) Energy distribution of the theoretical lowest $150^{+}$states and expectation value of the $f$-boson number operator $\left\langle\hat{n}_{f}\right\rangle$ for the considered Sm and Gd isotopes. Note that, concerning ${ }^{146} \mathrm{Sm}$ (a) and ${ }^{148} \mathrm{Gd}(\mathrm{g})$, the $0^{+}$states with an energy higher than $8 \mathrm{MeV}$ are not shown.

many other $0^{+}$states are also formed by the coupling of positive- and negative-parity (octupole) bosons. For both $\mathrm{Sm}$ and $\mathrm{Gd}$ chains, the $0^{+}$states become more populated in the lower-energy region for the heavier isotopes, where the quadrupole-octupole coupling becomes more enhanced. Particularly in the $\mathrm{Gd}$ isotopes, the level scheme for the $0^{+}$ states becomes most compressed around ${ }^{152} \mathrm{Gd}$ [panel (i)] or

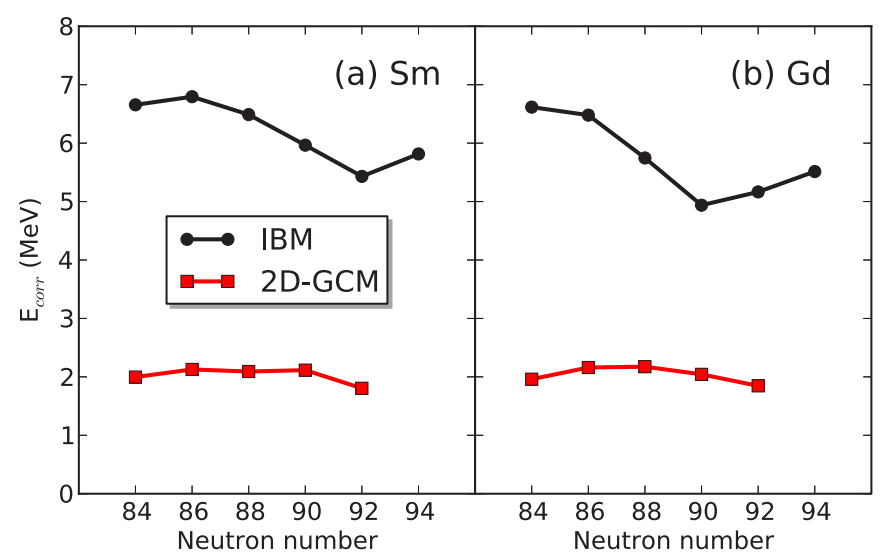

FIG. 12. (Color online) The correlation energies obtained from the IBM and the two-dimensional (2D) GCM for ${ }^{146-156} \mathrm{Sm}$ and ${ }^{148-158} \mathrm{Gd}$ isotopes.
${ }^{154} \mathrm{Gd}$ [panel (j)], where the corresponding PES is noticeably soft in both $\beta_{2}$ and $\beta_{3}$ deformations [see Figs. 1(i) and 1(j)].

\section{Correlation energy}

In this section, we discuss the correlation energies defined as $[19,50]$

$$
E_{\mathrm{Corr}}=E_{\mathrm{HFB}}^{\mathrm{g.s.}}-E\left(0_{1}^{+}\right)
$$

where $E_{\mathrm{HFB}}^{\text {g.s. }}$ represents the HFB ground-state energy and $E\left(0_{1}^{+}\right)$the one for the $0_{1}^{+}$state. The IBM correlation energies are depicted in Fig. 12 together with the ones obtained in previous two-dimensional Gogny-D1M GCM calculations. Results are shown in panel (a) for ${ }^{146-156} \mathrm{Sm}$ and in panel (b) for ${ }^{148-158} \mathrm{Gd}$. Though the correlation energies are different in both approaches, the largest values of $E_{\text {Corr }}$ are obtained for the lighter nuclei with $N \leqslant 88$ which are rather soft in the $\beta_{2}$ and $\beta_{3}$ degrees of freedom. This confirms that correlations beyond the mean-field approach can become significant in soft nuclear systems.

\section{SUMMARY}

In summary, we have carried out spectroscopic calculations aimed to describe the quadrupole and octupole collective 
states in $\mathrm{Sm}$ and $\mathrm{Gd}$ isotopes. Our starting point was a set of $Q_{20}-Q_{30}$ constrained HFB calculations, with the D1M parametrization of the Gogny effective interaction, used to produce a PES. This PES is then used to obtain the parameters of an IBM Hamiltonian including $s, d$, and $f$ bosons. Spectral properties of both positive- and negative-parity states associated with the reflection symmetric and asymmetric shapes, respectively, are obtained after diagonalization of the IBM Hamiltonian. The parameters of the IBM Hamiltonian are determined by mapping the Gogny-HFB mean-field energy surface onto the corresponding energy expectation value of the boson condensate state.

The systematics of the energy spectra and transition rates, associated with both positive- and negative-parity yrast states, points to the onset of notable octupole correlation around $N \approx 88$, characterized by the $\beta_{3}$-soft energy surfaces (Fig. 1) and the corresponding negative-parity band lowering in energy with respect to the positive-parity ground-state band (Fig. 5). From $N \geqslant 90$ on, the PES no longer exhibits $\beta_{3}$ softness, and the corresponding negative-parity band is pushed up in energy with respect to the ground-state band. The mean-field $\beta_{2} \beta_{3}$ energy surface (Fig. 1), the derived parameters in the $s d f$ Hamiltonian (Fig. 3), the resultant energy levels (Figs. 4 and 5), and transition rates (Fig. 8) correlate very well with each other in systematics with the number of valence nucleons. In addition, the spectroscopic properties resulting from the model turn out to be generally in reasonable agreement with the systematics of the available experimental data, and also to be consistent with the previous GCM calculation (Figs. 6 and 8) starting from the common Gogny parametrization D1M [19].

However, an in-depth analysis of the energy spectra and the $E 2$ and $E 1$ transition rates in the two characteristic cases, $\beta_{2}$ and $\beta_{3}$-soft nucleus ${ }^{150} \mathrm{Sm}$ and strongly $\beta_{2}$ deformed nucleus ${ }^{158} \mathrm{Gd}$, has revealed that an improvement of the model is required so as to give a better description not only of the yrast states but also of the nonyrast states. For example, our model in its current version is not able to describe well the bandhead of side bands, particularly that of the $\beta$-vibrational $\left(K^{\pi}=0_{2}^{+}\right)$band (Figs. 9 and 10$)$. A possible reason could be that the model space used for the present work might be rather limited to handle such a complex nuclear structure. This would require the extension of our model space to include configuration mixing specific to the intruder state and/or to introduce triaxial degrees of freedom. In addition, the model has failed in reproducing some of the $E 1$ properties, especially for those associated with the states in nonyrast negative-parity bands (Fig. 10). Several solutions have been proposed that could help to fix the problem: extension of the $E 1$ operator to include higher-order terms; explicit inclusion of $p$ boson in the model space. Improving the description of these properties will be a topic of future study. Significance of the $p$-boson effect in the $E 1$ excitation observed in rare-earth nuclei has been addressed in Ref. [83], though in the different context of $\alpha$ clustering.

We have also analyzed the wave-function content of some lower-lying excited $0^{+}$states for the considering nuclei and found that in many of the nuclei considered, the $0_{2}^{+}$states can be the consequence of the coupling of two-octupole phonons. This could be a possible explanation for the large number of low-energy excited $0^{+}$states found in rare-earth nuclei.

\section{ACKNOWLEDGMENTS}

K.N. acknowledges the support by the Marie Curie Actions grant within the Seventh Framework Program of the European Commission under Grant No. PIEF-GA-2012-327398. The work of L.M.R. is supported in part by Spanish MINECO Grants No. FPA2012-34694 and No. FIS2012-34479 and by the Consolider-Ingenio 2010 program MULTIDARK Grant No. CSD2009-00064.
[1] P. A. Butler and W. Nazarewicz, Rev. Mod. Phys. 68, 349 (1996).

[2] P. A. Butler and W. Nazarewicz, Nucl. Phys. A 533, 249 (1991).

[3] L. P. Gaffney, P. A. Butler, M. Scheck, A. B. Hayes, F. Wenander, M. Albers, B. Bastin, C. Bauer, A. Blazhev, S. Bönig et al. Nature (London) 497, 199 (2013).

[4] W. Urban, R. Lieder, W. Gast, G. Hebbinghaus, A. KrämerFlecken, K. Blume, and H. Hübel, Phys. Lett. B 185, 331 (1987).

[5] W. Urban, R. Lieder, J. Bacelar, P. Singh, D. Alber, D. Balabanski, W. Gast, H. Grawe, G. Hebbinghaus, J. Jongman et al., Phys. Lett. B 258, 293 (1991).

[6] P. E. Garrett, W. D. Kulp, J. L. Wood, D. Bandyopadhyay, S. Choudry, D. Dashdorj, S. R. Lesher, M. T. McEllistrem, M. Mynk, J. N. Orce et al., Phys. Rev. Lett. 103, 062501 (2009).

[7] R. F. Casten and N. V. Zamfir, Phys. Rev. Lett. 87, 052503 (2001).

[8] F. Iachello, Phys. Rev. Lett. 87, 052502 (2001).

[9] S. R. Lesher, A. Aprahamian, L. Trache, A. Oros-Peusquens, S. Deyliz, A. Gollwitzer, R. Hertenberger, B. D. Valnion, and G. Graw, Phys. Rev. C 66, 051305 (2002).

[10] N. V. Zamfir, J.-y. Zhang, and R. F. Casten, Phys. Rev. C 66, 057303 (2002).
[11] S. Marcos, H. Flocard, and P. H. Heenen, Nucl. Phys. A 410, 125 (1983).

[12] W. Nazarewicz, P. Olanders, I. Ragnarsson, J. Dudek, G. A. Leander, P. Möller, and E. Ruchowsa, Nucl. Phys. A 429, 269 (1984).

[13] W. Nazarewicz and P. Olanders, Nucl. Phys. A 441, 420 (1985).

[14] P. Bonche, P.-H. Heenen, H. Flocard, and D. Vautherin, Phys. Lett. B 175, 387 (1986).

[15] P. Bonche, in The Variation of Nuclear Shapes, edited by J. D. Garrett (World Scientific, Singapore, 1988), p. 302.

[16] J. L. Egido and L. M. Robledo, Nucl. Phys. A 524, 65 (1991).

[17] L. M. Robledo, M. Baldo, P. Schuck, and X. Viñas, Phys. Rev. C 81, 034315 (2010).

[18] L. M. Robledo and G. F. Bertsch, Phys. Rev. C 84, 054302 (2011).

[19] R. Rodríguez-Guzmán, L. M. Robledo, and P. Sarriguren, Phys. Rev. C 86, 034336 (2012).

[20] L. M. Robledo and P. A. Butler, Phys. Rev. C 88, 051302 (2013).

[21] O. Scholten, F. Iachello, and A. Arima, Ann. Phys. (NY) 115, 325 (1978).

[22] J. Engel and F. Iachello, Nucl. Phys. A 472, 61 (1987). 
[23] T. Otsuka and M. Sugita, Phys. Lett. B 209, 140 (1988).

[24] D. Kusnezov and F. Iachello, Phys. Lett. B 209, 420 (1988).

[25] P. D. Cottle and N. V. Zamfir, Phys. Rev. C 58, 1500 (1998).

[26] P. G. Bizzeti and A. M. Bizzeti-Sona, Phys. Rev. C 70, 064319 (2004).

[27] D. Bonatsos, D. Lenis, N. Minkov, D. Petrellis, and P. Yotov, Phys. Rev. C 71, 064309 (2005).

[28] D. Lenis and D. Bonatsos, Phys. Lett. B 633, 474 (2006).

[29] P. G. Bizzeti and A. M. Bizzeti-Sona, Phys. Rev. C 77, 024320 (2008).

[30] P. G. Bizzeti and A. M. Bizzeti-Sona, Phys. Rev. C 81, 034320 (2010).

[31] R. V. Jolos, P. von Brentano, and J. Jolie, Phys. Rev. C 86, 024319 (2012).

[32] N. Minkov, S. Drenska, M. Strecker, W. Scheid, and H. Lenske, Phys. Rev. C 85, 034306 (2012).

[33] P. G. Bizzeti and A. M. Bizzeti-Sona, Phys. Rev. C 88, 011305 (2013).

[34] F. Iachello and A. D. Jackson, Phys. Lett. B 108, 151 (1982).

[35] H. J. Daley and F. Iachello, Ann. Phys. (NY) 167, 73 (1986).

[36] T. M. Shneidman, G. G. Adamian, N. V. Antonenko, R. V. Jolos, and W. Scheid, Phys. Lett. B 526, 322 (2002).

[37] W. Zhang, Z. P. Li, S. Q. Zhang, and J. Meng, Phys. Rev. C 81, 034302 (2010).

[38] B.-N. Lu, E.-G. Zhao, and S.-G. Zhou, Phys. Rev. C 85, 011301 (2012).

[39] B.-N. Lu, J. Zhao, E.-G. Zhao, and S.-G. Zhou, Phys. Rev. C 89, 014323 (2014).

[40] M. Bender, P.-H. Heenen, and P.-G. Reinhard, Rev. Mod. Phys. 75, 121 (2003).

[41] T. H. R. Skyrme, Nucl. Phys. 9, 615 (1958).

[42] D. Vautherin and D. M. Brink, Phys. Rev. C 5, 626 (1972).

[43] J. Decharge, M. Girod, and D. Gogny, Phys. Lett. B 55, 361 (1975).

[44] D. Vretenar, A. V. Afanasjev, G. Lalazissis, and P. Ring, Phys. Rep. 409, 101 (2005).

[45] T. Nikšić, D. Vretenar, and P. Ring, Prog. Part. Nucl. Phys. 66, 519 (2011).

[46] R. Rodríguez-Guzmán, J. L. Egido, and L. M. Robledo, Nucl. Phys. A 709, 201 (2002).

[47] P. Ring and P. Schuck, The Nuclear Many-body Problem (Springer-Verlag, Berlin, 1980).

[48] L. M. Robledo and R. R. Rodrguez-Guzmán, J. Phys. G: Nucl. Part. Phys. 39, 105103 (2012).

[49] K. Nomura, N. Shimizu, and T. Otsuka, Phys. Rev. Lett. 101, 142501 (2008).

[50] K. Nomura, N. Shimizu, and T. Otsuka, Phys. Rev. C 81, 044307 (2010).

[51] K. Nomura, T. Otsuka, N. Shimizu, and L. Guo, Phys. Rev. C 83, 041302 (2011).

[52] K. Nomura, N. Shimizu, D. Vretenar, T. Nikšić, and T. Otsuka, Phys. Rev. Lett. 108, 132501 (2012).

[53] K. Nomura, R. Rodríguez-Guzmán, L. M. Robledo, and N. Shimizu, Phys. Rev. C 86, 034322 (2012).
[54] K. Nomura, D. Vretenar, and B.-N. Lu, Phys. Rev. C 88, 021303 (2013).

[55] K. Nomura, D. Vretenar, T. Nikšić, and B.-N. Lu, Phys. Rev. C 89, 024312 (2014)

[56] S. Goriely, S. Hilaire, M. Girod, and S. Péru, Phys. Rev. Lett. 102, 242501 (2009).

[57] R. Rodríguez-Guzmán, P. Sarriguren, L. M. Robledo, and S. Perez-Martin, Phys. Lett. B 691, 202 (2010).

[58] R. Rodríguez-Guzmán, P. Sarriguren, and L. M. Robledo, Phys. Rev. C 82, 044318 (2010).

[59] R. Rodríguez-Guzmán, P. Sarriguren, and L. M. Robledo, Phys. Rev. C 82, 061302 (2010).

[60] S. A. Giuliani, L. M. Robledo, and R. Rodríguez-Guzmán, Phys. Rev. C 90, 054311 (2014).

[61] J. F. Berger, M. Girod, and D. Gogny, Nucl. Phys. A 428, 23 (1984).

[62] We equally use the multipole moment values $Q_{l 0}$ and deformation parameters $\beta_{l}$ to talk about deformation.

[63] T. Otsuka, A. Arima, and F. Iachello, Nucl. Phys. A 309, 1 (1978).

[64] M. Sugita, T. Otsuka, and P. von Brentano, Phys. Lett. B 389, 642 (1996).

[65] A. F. Barfield, B. R. Barrett, J. L. Wood, and O. Scholten, Ann. Phys. 182, 344 (1988).

[66] J. N. Ginocchio and M. W. Kirson, Nucl. Phys. A 350, 31 (1980).

[67] N. V. Zamfir and D. Kusnezov, Phys. Rev. C 63, 054306 (2001)

[68] M. Babilon, N. V. Zamfir, D. Kusnezov, E. A. McCutchan, and A. Zilges, Phys. Rev. C 72, 064302 (2005).

[69] D. J. Thouless and J. G. Valatin, Nucl. Phys. 31, 211 (1962).

[70] H. Schaaser and D. M. Brink, Nucl. Phys. A 452, 1 (1986).

[71] D. Kusnezov, computer program OCTUPOLE (unpublished).

[72] W. Long, J. Meng, N. Van Giai, and S.-G. Zhou, Phys. Rev. C 69, 034319 (2004).

[73] T. Nikšić, D. Vretenar, and P. Ring, Phys. Rev. C 78, 034318 (2008).

[74] T. Otsuka, Phys. Rev. Lett. 46, 710 (1981).

[75] T. Otsuka and J. N. Ginocchio, Phys. Rev. Lett. 55, 276 (1985).

[76] F. Iachello and A. Arima, The Interacting Boson Model (Cambridge University Press, Cambridge, UK, 1987).

[77] Brookhaven National Nuclear Data Center, http://www. nndc.bnl.gov

[78] F. R. Metzger, Phys. Rev. C 14, 543 (1976).

[79] T. Kibédi and R. H. Spear, At. Data Nucl. Data Tables 80, 35 (2002).

[80] H. Pitz, U. Berg, R. Heil, U. Kneissl, R. Stock, C. Wesselborg, and P. V. Brentano, Nucl. Phys. A 492, 411 (1989).

[81] T. Otsuka, Phys. Lett. B 182, 256 (1986).

[82] D. Meyer, V. Wood, R. Casten, C. Fitzpatrick, G. Graw, D. Bucurescu, J. Jolie, P. von Brentano, R. Hertenberger, H.-F. Wirth et al., Phys. Lett. B 638, 44 (2006).

[83] M. Spieker, S. Pascu, A. Zilges, and F. Iachello, Phys. Rev. Lett. 114, 192504 (2015). 